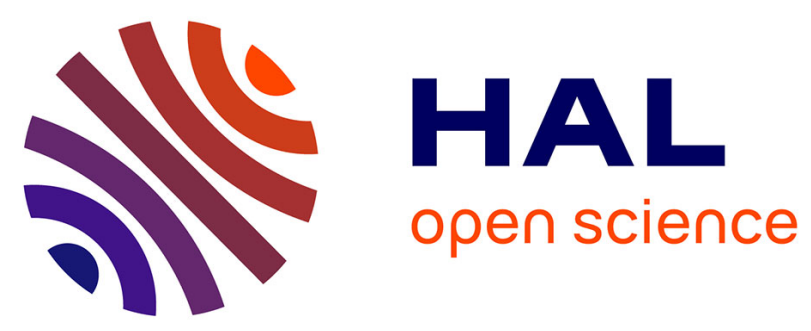

\title{
Surrogate data methods based on a shuffling of the trials for synchrony detection: the centering issue
}

Mélisande Albert, Yann Bouret, Magalie Fromont, Patricia Reynaud-Bouret

\section{To cite this version:}

Mélisande Albert, Yann Bouret, Magalie Fromont, Patricia Reynaud-Bouret. Surrogate data methods based on a shuffling of the trials for synchrony detection: the centering issue. Neural Computation, 2016, 28 (1), pp.2352-2392. 10.1162/NECO_a_00839. hal-01154918v2

\section{HAL Id: hal-01154918 \\ https://hal.science/hal-01154918v2}

Submitted on 12 Jul 2016

HAL is a multi-disciplinary open access archive for the deposit and dissemination of scientific research documents, whether they are published or not. The documents may come from teaching and research institutions in France or abroad, or from public or private research centers.
L'archive ouverte pluridisciplinaire $\mathbf{H A L}$, est destinée au dépôt et à la diffusion de documents scientifiques de niveau recherche, publiés ou non, émanant des établissements d'enseignement et de recherche français ou étrangers, des laboratoires publics ou privés. 


\section{Surrogate data methods based on a shuffling of the trials for synchrony detection: the centering is- sue}

\section{Mélisande Albert $^{1}$}

Melisande.Albert@unice.fr

Yann Bouret $^{2}$

Yann.Bouret@unice.fr

Magalie Fromont ${ }^{3}$

magalie.fromont@univ-rennes2.fr

\section{Patricia Reynaud-Bouret ${ }^{1}$}

reynaudb@unice.fr

${ }^{1}$ Univ. Nice Sophia Antipolis, CNRS, LJAD, UMR 7351, 06100 Nice, France.

${ }^{2}$ Univ. Nice Sophia Antipolis, CNRS, LPMC, UMR 7336, 06100 Nice, France.

${ }^{3}$ Univ. Européenne de Bretagne, CNRS, IRMAR, UMR 6625, 35043 Rennes Cedex, France.

Keywords: Unitary Events - Synchronization - Independence tests - Trial-Shuffling - Permutation - Bootstrap - Point process - Multiple testing 


\section{Abstract}

We investigate several distribution free dependence detection procedures, all based on a shuffling of the trials, from a statistical point of view. The mathematical justification of such procedures lies in the bootstrap principle and its approximation properties. In particular we show that such a shuffling has mainly to be done on centered quantities - that is quantities with zero mean under independence - to construct correct $p$-values, meaning that the corresponding tests control their False Positive (FP) rate. Thanks to this study, we introduce a method, named Permutation UE, which consists in a multiple testing procedure based on permutation of experimental trials and delayed coincidence count. Each involved single test of this procedure achieves the prescribed level, so that the corresponding multiple testing procedure controls the False Discovery Rate (FDR), and this with as few assumptions as possible on the underneath distribution, except independence and identical distribution across trials. The mathematical meaning of this assumption is discussed and it is in particular argued that it does not mean what is commonly referred in neuroscience to as cross-trials stationarity. Some simulations show moreover that Permutation UE outperforms the trial-shuffling of (Pipa \& Grün, 2003) and the MTGAUE method of (Tuleau-Malot et al., 2014) in terms of single levels and FDR, for a comparable amount of False Negatives. Application to real data is also provided. 


\section{Introduction}

The possible time dependence either between cerebral areas or between neurons, and in particular the synchrony phenomenon, has been vastly debated and investigated as a potential element of the neuronal code (Singer, 1993). To detect such a phenomenon at the microscopic level, multielectrodes are usually used to record the nearby electrical activity. After pretreatment, the time occurrences of action potentials (spikes) for several neurons are therefore available. One of the first steps of analysis is then to understand whether and how two simultaneously recorded spike trains, corresponding to two different neurons, are dependent or not.

Several methods have been used to detect synchrony (Perkel et al., 1967; Aertsen et al., 1989). Among the most popular ones, the Unitary Events (UE) method, due to Grün and collaborators (Grün, 1996; Grün et al., 2002a,b, 2010), has been applied in the last decade to a vast amount of real data (see, e.g., (Kilavik et al., 2009) and references therein). Two of its main features are at the root of its popularity: the UE method is not only able to give a precise location in time of the dependence periods, but also to quantify the degree of dependence by providing $p$-values for the independence tests.

One can decompose the method in three main steps:

(i) The first step consists in choosing a way to count coincidences. In the original UE method, the point processes modeling the data are binned and clipped at a rough level (see Figure 1.A for a more precise description), the bins being about $5 \mathrm{~ms}$ wide. However, it is proven in (Grün et al., 1999) that the binned coincidence count as a result of this preprocessing may induce a loss in synchrony detection of about $60 \%$ in certain cases (see also an illustration in Figure 1.A). The idea of (Grün et al., 1999) was 
therefore to keep the data at the initial resolution level despite its high dimension, but to define the notion of multiple shift (MS) coincidence count, nicely condensing the dependence feature that neurobiologists want to analyze without any loss in synchrony detection. The delayed coincidence count is a generalization of this notion to non discretized process and which still does not suffer from any loss in synchrony detection (see Figure 1.B). Other coincidence count notions have also been used such as the one in (Louis et al., 2010b) which also holds for non discretized process.

(ii) Once the coincidence count is fixed, one needs to understand what is the typical behavior of this quantity under independence, so that independence is rejected if the count is significantly unusual. To do so, the original method estimates the expected number of coincidence under independence and assumes a Poisson distribution of the count under independence. This assumption has been shown to be non completely adequate in (Pipa et al., 2013; Tuleau-Malot et al., 2014) and the plug-in of estimates of the underlying firing rates has also been discussed in (Gütig et al., 2001; Tuleau-Malot et al., 2014). Notably in (Gütig et al., 2001) another method is introduced which uses conditional distribution to avoid the misuse of plug-in estimates. However in all those works, very strong assumptions on the distribution of the spikes are made: either bins are assumed to be independent and identically distributed or the spike trains are assumed to be Poisson or at least renewal processes. However conclusive experimental evidence combined with many statistical and modeling studies show that those distribution assumptions are not realistic - see (Nawrot et al., 2008; Farkhooi et al., 2009; Pouzat \& Chaffiol, 2009; Avila-Akerberg \& Chacron, 2011) and the references therein. This is the reason why a lot of interest has been shown on surrogate data methods 
- see (Grün, 2009; Louis et al., 2010a) for a methodological review. These methods, unlike the ones cited above, are not linked to a particular coincidence count and they can be indifferently applied to any of the previous counts discussed above. The main idea is to use the original observed data set and to combine it with a computer random generator to produce new artificial data sets mimicking how the data set would behave under independence. Thanks to these surrogate data sets, it is a priori possible to estimate the distribution of the coincidence count under independence and therefore to build reasonable $p$-values. This can usually be achieved in practice through parallel programming and Monte-Carlo approximation (Louis et al., 2010a).

There are mainly two trends in surrogate data methods. Either the trials are shuffled (Pipa \& Grün, 2003; Pipa et al., 2003), but it has been shown that this method suffers from a non controlled False Positive rate when there is cross-trials non-stationarity (Grün et al, 2003); or the spikes themselves are slightly moved as in the dithering method - see (Louis et al., 2010b) and the references therein. This last method is more able to cope with cross-trials non-stationarity. Indeed, and even under cross-trials nonstationarity, several more or less technical variants of this method are able to reproduce the mean intensity, also called profile or rate, and even the interspike interval distribution. However those methods cannot mimic the whole distribution of the coincidence count under independence. As a consequence, the best dithering methods, in the sense that these methods are able to control their False Positive rate even for highly non homogeneous processes in time, are much too conservative, as assessed in (Louis et al., 2010b).

(iii) The third step of a UE method is linked to the multiple testing aspect, seen by 
S. Grün herself (Grün, 2009) as "a useful side-effect" of the fact that the original UE method needs homogeneity in time. Because of this drawback in the original procedure, the UE tests described above are performed on small sliding windows on which the homogeneity assumption is realistic. This allows, as a by-product, "a time resolved analysis [which] shows potential modulation of synchrony". It has been proved however in (Tuleau-Malot et al., 2014) that the procedure needs therefore to be corrected for the multiplicity of the tests with, for instance, Benjamini and Hochberg procedure to control the False Discovery Rate (Benjamini \& Hochberg, 1995).

We here focus on surrogate data methods based on a shuffling or resampling of the trials. Such procedures are mathematically justified by the bootstrap principle that indeed provides several ways to shuffle trials, all able to reproduce the distribution of the count under independence, if applied to centered quantities. So our main concern is to warn people using methods based on a shuffling of the trials against a direct application of these methods to rough coincidence counts, which are not correctly centered. As a consequence of this study, we show that a permutation of the trials in line with (Hoeffding, 1952; Romano, 1989; Romano \& Wolf , 2005) is the most able to mimic the correct distribution among the resampling approaches investigated here. We couple it with the delayed coincidence count to avoid loss in synchrony detection and a Benjamini and Hochberg procedure controlling the False Discovery Rate when considering sliding windows, to obtain a new method named Permutation UE. Because resampling methods are quite demanding in terms of computational cost, we also propose a fast algorithm to compute the delayed coincidence count, with a computational cost equivalent to the one of the binned coincidence count. 
A major assumption of the present work, due to the shuffling of the trials, is the independence and the identical distribution between trials in the probabilistic sense. However this mathematical notion does not mean stationarity across trials, as commonly expressed in the neuroscience literature (Arieli et al., 1996; Grün et al, 2003; Churchland et al., 2010; Nawrot, 2010; Farkhooi et al., 2011; Churchland et al., 2011; Litwin-Kumar \& Doiron, 2012; Farkhooi et al., 2013). This point is clearly discussed hereafter in Section 5.

We begin with describing the mathematical framework in Section 2, by giving the notation and the definition of binned and delayed coincidence counts together with a detailed fast algorithm to compute the delayed one. In Section 3, we precisely discuss the centering problem and its effect on the methods based on a shuffling of the trials. In Section 4, we detail the Permutation UE method and apply it to real data. In Section 5, we discuss the limit of the methods in terms of both distribution free aspects, and cross-trials stationarity and provide some open questions.

\section{Framework}

We start by giving some useful notation and reminders to understand the construction and discussion of the dependence detection methods using a shuffling of the trials.

\subsection{Notation}

In all the sequel, $X^{1}$ and $X^{2}$ denote two point processes modeling the spike trains of two simultaneously recorded neurons and $X$ represents the couple $\left(X^{1}, X^{2}\right)$. The ab- 
breviation "i.i.d." stands for independent and identically distributed. In this sense, by assuming that $n$ independent and identically distributed trials are observed, the observation is modeled by an i.i.d. sample of size $n$ of couples from the same distribution as $X$, meaning $n$ i.i.d. copies $X_{1}, \ldots, X_{n}$ of $X$. This sample is denoted in the sequel by $\mathbb{X}_{n}=\left(X_{1}, \ldots, X_{n}\right)$. The corresponding probability and expectation are respectively denoted by $\mathbb{P}$ and $\mathbb{E}$. For another random variable, $Y$, conditional probability and conditional expectation given $Y$ are respectively denoted $\mathbb{P}(\cdot \mid Y)$ and $\mathbb{E}[\cdot \mid Y]$, they both are random quantities that still depend on the value of $Y$.

The notation $\mathbf{1}_{X \in A}$ stands for a function whose value is 1 if $X$ belongs to $A$ and 0 otherwise. In particular note that

$$
\mathbb{P}(X \in A)=\mathbb{E}\left[\mathbf{1}_{X \in A}\right]=\mathbb{E}\left[\mathbb{E}\left[\mathbf{1}_{X \in A} \mid Y\right]\right]=\mathbb{E}[\mathbb{P}(X \in A \mid Y)]
$$

which amounts to integrate first in the conditional distribution of $X$ given $Y$ and then to integrate in the distribution of $Y$.

Since assessing dependence between $X^{1}$ and $X^{2}$ is the main focus of the present work, the following notation is useful: $X^{\Perp}$ denotes a couple $\left(X^{1, \Perp}, X^{2, \Perp}\right)$ such that $X^{1, \Perp}\left(\right.$ resp. $\left.X^{2, \Perp}\right)$ has the same distribution as $X^{1}$ (resp. $\left.X^{2}\right)$, but $X^{1, \Perp}$ is independent of $X^{2, \Perp}$. In particular, the couple $X^{\Perp}$ has the same marginals as the couple $X$. Moreover, $\mathbb{X}_{n}^{\Perp}=\left(X_{1}^{\Perp}, \ldots, X_{n}^{\Perp}\right)$, with $X_{i}^{\Perp}=\left(X_{i}^{1, \Perp}, X_{i}^{2, \Perp}\right)$, denotes an i.i.d. sample of size $n$ from the same distribution as $X^{\Perp}$, and $\mathbb{P}_{\Perp}$ and $\mathbb{E}_{\Perp}$ are the corresponding probability and expectation.

Note in particular that if the two observed neurons indeed behave independently, then the observed sample $\mathbb{X}_{n}$ has the same distribution as $\mathbb{X}_{n}^{\Perp}$.

Finally, for any point process $X^{j}(j=1,2), d N_{X^{j}}$ stands for its associated point 
measure, defined for all function $f$ by:

$$
\int f(u) d N_{X^{j}}(u)=\sum_{T \in X^{j}} f(T),
$$

and for any interval $I, N_{X^{j}}(I)$ denotes the number of points of $X^{j}$ observed in $I$.

\subsection{Binned and delayed coincidence counts}

Because of the way neurons transmit information through action potentials, it is commonly admitted that the dependence between the spike trains of two neurons is due to temporal correlations between spikes produced by both neurons. Informally, a coincidence occurs when two spikes (one from each neuron) appear with a delay less than a fixed $\delta$ (of the order of a few milliseconds). Several coincidence count functions have been defined in the neuroscience literature, and among them the classical binned coincidence count, introduced in (Grün et al., 2002a,b).

Definition 1 The binned coincidence count between point processes $X^{1}$ and $X^{2}$ on the interval $[a, b]$ with $b-a=M \delta$ for an integer $M \geq 2$ and a fixed delay $\delta>0$ is given by

$$
\psi_{\delta}^{\text {coinc }}\left(X^{1}, X^{2}\right)=\sum_{\ell=1}^{M} \mathbf{1}_{N_{X^{1}}\left(I_{\ell}\right) \geq 1} \mathbf{1}_{N_{X^{2}}\left(I_{\ell}\right) \geq 1},
$$

where $I_{\ell}$ is the $\ell$ th bin of length $\delta$, i.e. $[a+(\ell-1) \delta, a+\ell \delta)$.

More informally, the binned coincidence count is the number of bins that contain at least one spike of each spike trains, as one can see on Figure 1.A.

The binned coincidence count computation algorithm is usually performed on already binned and clipped data (see Figure 1). Therefore, given two sequences of 0 and 
1 of length $M=(b-a) \delta^{-1}$, the number of operations needed to compute the binned coincidence count is $O(M)=O\left((b-a) \delta^{-1}\right)$ (without taking the binning preprocessing into account).

The more recent notion of delayed coincidence count, introduced in (Tuleau-Malot et al., 2014), is a generalization of the multiple-shift coincidence count, defined in (Grün et al., 1999) for discretized point processes, to non necessarily discretized point processes.

Definition 2 The delayed coincidence count between point processes $X^{1}$ and $X^{2}$ on the interval $[a, b]$ is given by

$$
\varphi_{\delta}^{\text {coinc }}\left(X^{1}, X^{2}\right)=\int_{a}^{b} \int_{a}^{b} \mathbf{1}_{|u-v| \leq \delta} d N_{X^{1}}(u) d N_{X^{2}}(v)
$$

More informally, $\varphi_{\delta}^{\text {coinc }}\left(X^{1}, X^{2}\right)$ is the number of couples of spikes (one spike from $X^{1}$ and one from $X^{2}$ ) appearing in $[a, b]$ with delay at most equal to $\delta$. A visual example is given on Figure 1.B. Note in particular that two coincidences are discarded by the binned coincidence count on this particular example: one because of the clipping effect in the third bin and one because of the effect of adjacent bins in the seventh and eighth bins. Both of them are counted in the delayed coincidence count.

A rather naive algorithm to compute delayed coincidence count would test whether for any pair $(u, v)$ of a spike $\mathrm{u}$ in $X^{1}$ and a spike $v$ in $X^{2}$, the delay $|u-v|$ is less than $\delta$ and to count the number of hits. This would lead to an algorithm whose complexity is in the product of the number of points in each spike train. If one assumes both spike trains to be Poisson with intensity $\lambda_{1}$ and $\lambda_{2}$, this algorithm has an average cost of order $O\left(\lambda_{1} \lambda_{2}(b-a)^{2}\right)$ and is therefore quadratic in the length of the interval. One can 
actually drastically improve this rate thanks to the following algorithm for which the result $c:=\varphi_{\delta}^{\text {coinc }}\left(X^{1}, X^{2}\right)$ is the delayed coincidence count.

\section{Delayed coincidence count algorithm}

Given two sequences $x_{1}$ and $x_{2}$ of ordered points with respective lengths $n_{1}=$ $N_{X^{1}}([a, b])$ and $n_{2}=N_{X^{2}}([a, b])$, representing the observations of two point processes $X^{1}$ and $X^{2}$,

- Initialize $j=1$ and $c=0$.

- For $i=1, \ldots, n_{1}$,

1. Assign $x_{l o w}=x_{1}[i]-\delta$.

2. While $j \leq n_{2}$ and $x_{2}[j]<x_{l o w}, j=j+1$.

3. If $j>n_{2}$, stop.

4. Else (here necessarily, $x_{2}[j] \geq x_{\text {low }}$ ),

4.a Assign $x_{u p}=x_{1}[i]+\delta$ and $k=j$.

4.b While $k \leq n_{2}$ and $x_{2}[k] \leq x_{u p}, c=c+1$ and $k=k+1$.

This algorithm is slightly more intricate but the computational complexity is much smaller than the previous one. Figure 1.C gives a visualization of the algorithm on a very simple example. The main point is that the index $j$ in Step 2 cannot decrease and therefore it is not making a double full loop on all the indices of both sequences $x_{1}$ and $x_{2}$. A pseudo double loop is made thanks to the index $k$ in step 4.b which indeed can take several times the same value but whose range is only governed by the number of points that appear in an interval of length $2 \delta$, namely $\left[x_{l o w}, x_{u p}\right]$, which is usually much smaller than the total length of the sequence $x_{2}$. More precisely, the complexity of the algorithm is therefore upper bounded, up to a constant, by $n_{1}$ (for steps 1,3 and 4.a), plus $n_{2}$ (for all steps 2 on all points of $x_{1}$, that is the range of 
the index $j$ which never decreases), and plus $n_{1}$ times the number of points of $x_{2}$ in a segment (namely $\left[x_{l o w}, x_{u p}\right]$ ) of length $2 \delta$ (for step 4.b). On average, if $X^{1}$ and $X^{2}$ are for instance independent homogeneous Poisson processes of respective intensities $\lambda_{1}$ and $\lambda_{2}$, the complexity is of order $O\left(\left(\lambda_{1}+\lambda_{2}+\lambda_{1} \lambda_{2} \delta\right)(b-a)\right)$. As compared with the binned coincidence count algorithm, whose complexity is of order $O\left(\delta^{-1}(b-a)\right)$, the present delayed coincidence count algorithm is therefore advantageous as soon as $\lambda_{1} \delta<<1$ and $\lambda_{2} \delta<<1$, conditions that are usually satisfied in practice (take for instance $\lambda_{1}=\lambda_{2}=30 \mathrm{~Hz}$ and $\delta=0.005 \mathrm{~s}$, which gives $\left.\lambda_{1} \delta=\lambda_{2} \delta=0.15\right)$. Even if both algorithms are linear in $(b-a)$, the delayed coincidence count algorithm exploits the sparsity of the spike trains through the constant $\left(\lambda_{1}+\lambda_{2}+\lambda_{1} \lambda_{2} \delta\right)$ in its complexity, instead of $\delta^{-1}$ in the complexity of the binned coincidence count algorithm. In Figure 1 is given a more visual representation of this sparsity: notice for instance that the bins with 0's do not even have to be taken into account in the present delayed coincidence count algorithm.

As explained in the introduction - point (ii) - all surrogate data methods (see (Louis et al., 2010a)) could in principle be applied to this notion of delayed coincidence count, at least when only two neurons are involved. In the sequel and for illustration purpose in the simulations, we apply the different surrogate methods of trial-shuffling type to the delayed coincidence count but the mathematical justification (Albert et al., 2015) and therefore the described behaviors in Section 3 are the same whatever the coincidence count that one would like to consider. 

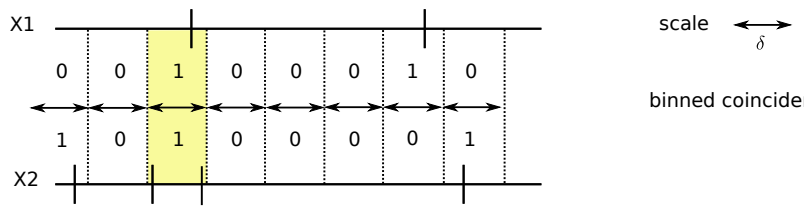

binned coincidence count $=1$

B: Delayed coincidence count

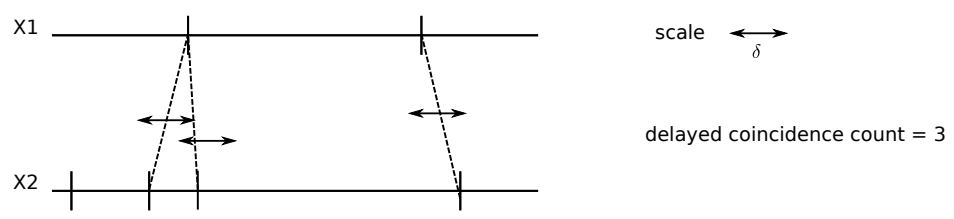

C: Algorithm for the delayed coincidence count

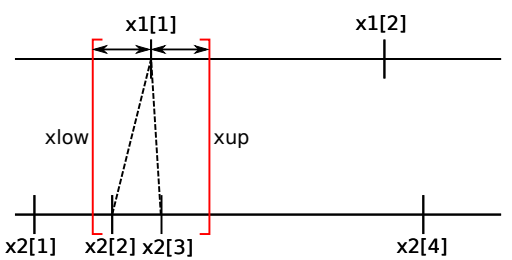

$\mathbf{i}=\mathbf{1}$, step 1 : position of xlow step $2: \mathrm{j}=1 \rightarrow 2$ step $3:$ no stop

step 4a: position of xup

step $4 \mathrm{~b}: \mathrm{k}=2 \rightarrow 4 ; \mathrm{c}=0 \rightarrow 2$

2 coincidences have been found for $x 1[1]$

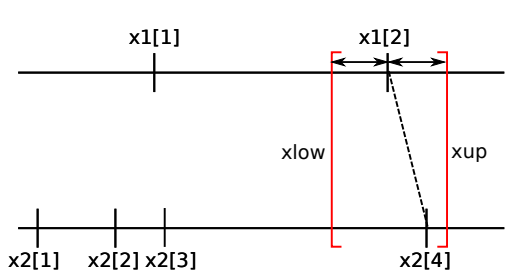

$\mathbf{i}=\mathbf{2}$, step 1 : new position of xlow

step $2: \mathrm{j}=2 \rightarrow 4$

step 3 : no stop

step 4a: new position of xup step $4 \mathrm{~b}: \mathrm{k}=4 \rightarrow 5 ; \mathrm{c}=2 \rightarrow 3$

1 coincidence has been found for $x 1[2]$

TOTAL : $c=3$ coincidences

Figure 1: Coincidence counts. Part A gives an example of binned coincidence count on a couple of spike trains $\left(X^{1}, X^{2}\right)$ (the spikes corresponding to the respective dashes on the line): after binning the data into blocks of length $\delta$, one only keeps the information whether there is at least a spike or not in the bin (clipping). The binned coincidence count is then the number of times there is a "1" for each spike train in the same bin. Part $\mathbf{B}$ gives on the same example the number of delayed coincidence count, that is the number of pairs of points (one on each spike train) at distance less than $\delta$. Note that these two coincidence counts are on this particular example different. Part $\mathbf{C}$ provides a visualization of the first steps of the proposed algorithm. In particular, note that it exploits the sparsity of the data represented via the vector $x_{1}$ and $x_{2}$ : there is no computational time spent on the central part of the drawing corresponding to the 0 's of the binned process. 


\section{Bootstrap and centering issues}

Given an interval of time $[a, b]$ and the observation of a sample $\mathbb{X}_{n}=\left(X_{1}, \ldots, X_{n}\right)$ corresponding to $n$ different trials in this interval, we focus here on the problem of testing the null hypothesis:

$$
\left(H_{0}\right) " X^{1} \text { and } X^{2} \text { are independent on }[a, b] "
$$

against:

$$
\left(H_{1}\right) " X^{1} \text { and } X^{2} \text { are not independent on }[a, b] " .
$$

All existing UE methods are based on the total number of coincidences:

$$
\mathbf{C}=\mathbf{C}\left(\mathbb{X}_{n}\right)=\sum_{i=1}^{n} \varphi\left(X_{i}^{1}, X_{i}^{2}\right)
$$

where $\varphi$ generically denotes either $\psi_{\delta}^{\text {coinc }}$, or $\varphi_{\delta}^{\text {coinc }}$, or other coincidence count functions that practitioners would like to use (see (Albert et al., 2015) for other choices). To underline what is observed or not, when $\mathrm{C}$ is computed on the observation of $\mathbb{X}_{n}$, it is denoted by $\mathbf{C}^{o b s}$, the total number of observed coincidences.

In the following, several of these UE methods are described, which all rely on the same paradigm: "reject $\left(H_{0}\right)$ when $\mathbf{C}^{o b s}$ is significantly different from what is expected under $\left(H_{0}\right)$ ". More precisely, the independence null hypothesis $\left(H_{0}\right)$ is rejected and the dependence is detected when a quantity, based on the difference between the observed coincidence count and what is expected under $\left(H_{0}\right)$, is smaller or larger than some critical values. Those critical values are obtained in various ways, each of them being peculiar to each method. 


\subsection{Importance of a centering step when parameters are unknown}

Before explaining the various resampling methods based on a shuffling of the trials investigated here where the centering issue appears as a major point, we want to underline that such a centering issue also occurs in more naive methods, for which this problem is easier to understand. Informally, there is a centering issue if a method is able to reproduce the distribution of centered quantities (that is with zero mean under independence) but is not able to do so for non centered quantities.

Let us first look at a toy example. If the values of the expectation and the variance of $\mathbf{C}$ under $\left(H_{0}\right)$, that is

$$
c_{0}=\mathbb{E}_{\Perp}[\mathbf{C}] \text { and } v_{0}=\mathbb{E}_{\Perp}\left[\left(\mathbf{C}-c_{0}\right)^{2}\right],
$$

are precisely known, then the classical central limit theorem gives under independence that

$$
\frac{\mathbf{C}\left(\mathbb{X}_{n}^{\Perp}\right)-c_{0}}{\sqrt{v_{0}}} \underset{n \rightarrow \infty}{\stackrel{\mathcal{L}}{\longrightarrow}} \mathcal{N}(0,1) .
$$

This means in particular that when the number of trials $n$ tends to infinity, the cumulative distribution function and the quantiles of $\left(\mathbf{C}\left(\mathbb{X}_{n}^{\Perp}\right)-c_{0}\right) / \sqrt{v_{0}}$ are tending to the ones of a standard Gaussian distribution, $\mathcal{N}(0,1)$, that is a Gaussian variable of mean 0 and variance 1 . Then, given $\alpha$ in $(0,1)$, the test which consists in rejecting $\left(H_{0}\right)$ when $\left(\mathbf{C}^{\text {obs }}-c_{0}\right) / \sqrt{v_{0}}$ is larger than $z_{1-\alpha}$, the $1-\alpha$ quantile of a standard Gaussian distribution, is asymptotically (in $n$, the number of trials) of False Positive (FP) rate $\alpha$. It means that, for this test, the probability of rejecting independence, whereas independence holds, is asymptotically equal to the prescribed $\alpha$.

In this particular case, we could rewrite the above procedure in a complete equiv- 
alent way, as follows: we reject the independence $\left(H_{0}\right)$ when $\mathbf{C}^{\text {obs }}$ is larger than the $1-\alpha$ quantile of $\mathcal{N}\left(c_{0}, v_{0}\right)$, Gaussian distribution of mean $c_{0}$ and variance $v_{0}$. Another way to state this is that as long as $c_{0}$ and $v_{0}$ are known, approximating the distribution of $\left(\mathbf{C}\left(\mathbb{X}_{n}^{\Perp}\right)-c_{0}\right) / \sqrt{v_{0}}$ by $\mathcal{N}(0,1)$ or approximating the distribution of $\mathbf{C}\left(\mathbb{X}_{n}^{\Perp}\right)$ by $\mathcal{N}\left(c_{0}, v_{0}\right)$ is completely equivalent: this is due to the scaling and shifting properties of the Gaussian distributions.

However, if $c_{0}$ and $v_{0}$ are unknown, and it is always the case in practice even if one assumes Poissonian spike trains (since the firing rates are unknown), one would like to replace $c_{0}$ and $v_{0}$ by estimates, namely $\hat{c}_{0}$ and $\hat{v}_{0}$ and proceed as previously. It has been shown in (Tuleau-Malot et al., 2014) that we cannot do that. Indeed the plugin step which consists in estimating the distribution of $\mathbf{C}\left(\mathbb{X}_{n}^{\Perp}\right)$ by $\mathcal{N}\left(\hat{c}_{0}, \hat{v}_{0}\right)$ instead of $\mathcal{N}\left(c_{0}, v_{0}\right)$ does not work for the non centered quantity C. Only the Gaussian approximation of the distribution of the centered quantity, namely $\mathbf{C}\left(\mathbb{X}_{n}^{\Perp}\right)-\hat{c}_{0}$, holds and at the price of modified variance. Note that this plug-in issue is known in different terms since (Gütig et al., 2001), who advertise for the use of conditional distribution. However both (Tuleau-Malot et al., 2014) and (Gütig et al., 2001) still assume strong distribution assumption (such as Poissonian features) that can be avoided by surrogate data methods. Can we show similar Gaussian approximations without such strong distribution assumptions?

Firstly it is possible to estimate $c_{0}$ without making any strong distribution assumption besides the fact that the trials are assumed to be i.i.d.

Indeed note that

$$
c_{0}=\mathbb{E}_{\Perp}\left[\sum_{i=1}^{n} \varphi\left(X_{i}^{1, \Perp}, X_{i}^{2, \Perp}\right)\right]=n \mathbb{E}_{\Perp}\left[\varphi\left(X^{1, \Perp}, X^{2, \Perp}\right)\right],
$$


and that for $i \neq i^{\prime}$, since $X_{i}$ is always assumed to be independent of $X_{i^{\prime}}$, the following equality holds

$$
\mathbb{E}\left[\varphi\left(X_{i}^{1}, X_{i^{\prime}}^{2}\right)\right]=\mathbb{E}_{\Perp}\left[\varphi\left(X^{1, \Perp}, X^{2, \Perp}\right)\right]
$$

Thus, $c_{0}$ can always be estimated in a distribution free manner by

$$
\hat{\mathbf{C}}_{0}\left(\mathbb{X}_{n}\right)=\frac{1}{n-1} \sum_{i \neq i^{\prime}} \varphi\left(X_{i}^{1}, X_{i^{\prime}}^{2}\right)
$$

The centered quantity of interest, in the sense that it has zero mean under independence, is therefore the difference:

$$
\mathbf{U}=\mathbf{U}\left(\mathbb{X}_{n}\right)=\mathbf{C}\left(\mathbb{X}_{n}\right)-\hat{\mathbf{C}}_{0}\left(\mathbb{X}_{n}\right)
$$

its observed version being denoted by $\mathbf{U}^{o b s}$.

The next step is to give the asymptotic distribution of $\mathbf{U}$ (or a renormalized version of it) without making any distribution assumptions in the same spirit as (1) so that one has access to quantiles and critical values. The main mathematical difficulty is that now $\hat{\mathbf{C}}_{0}\left(\mathbb{X}_{n}\right)$ is random and that therefore $\mathbf{U}$ is not a simple sum over all the trials, but a sum on all the $\left(i, i^{\prime}\right)$ pairs of trials 1 .

Nevertheless, some asymptotic theorems close in spirit to central limit theorems and proven in (Albert et al., 2015), show that under mild conditions (always satisfied in practice in the present cases) the following convergence result holds:

$$
\mathbf{Z}\left(\mathbb{X}_{n}^{\Perp}\right)=\frac{\mathbf{U}\left(\mathbb{X}_{n}^{\Perp}\right)}{\sqrt{n} \hat{\sigma}\left(\mathbb{X}_{n}^{\Perp}\right)} \underset{n \rightarrow \infty}{\stackrel{\mathcal{L}}{\longrightarrow}} \mathcal{N}(0,1)
$$

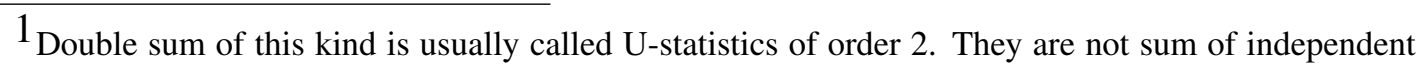
variables because of the double sum.
} 
where

$$
\hat{\sigma}^{2}\left(\mathbb{X}_{n}\right)=\frac{4}{n(n-1)(n-2)} \sum_{i, j, k \text { all different }} h\left(X_{i}, X_{j}\right) h\left(X_{i}, X_{k}\right),
$$

with

$$
h(x, y)=\frac{1}{2}\left[\varphi\left(x^{1}, x^{2}\right)+\varphi\left(y^{1}, y^{2}\right)-\varphi\left(x^{1}, y^{2}\right)-\varphi\left(y^{1}, x^{2}\right)\right]
$$

This result means that one exactly has a distribution approximation of the same form as the one of the toy example (1).

As above for the toy example, denoting by $\mathbf{Z}^{\text {obs }}$ the quantity $\mathbf{Z}$ computed on the observed sample, (4) implies that for some fixed $\alpha$ in $(0,1)$, the test that consists in rejecting $\left(H_{0}\right)$ when $\mathbf{Z}^{\text {obs }} \geq z_{1-\alpha}$, is asymptotically of level $\alpha$.

Let us look more closely at the quality of the approximation (4) on Figure 2. Clearly, one can see that the distribution approximation is good when $n$ is large $(n=200)$ as expected, but not so convincing for small values of $n(n=20$, or even $n=50)$, particularly in the tails of the distributions. However, as it is especially the tails of the distributions that are involved in the test through the quantile $z_{1-\alpha}$, one can wonder, by looking at Figure 2, if it may perform reasonably well in practice with a usual number of a few tens of trials.

However, unlike the toy example and in line with what happens in (Tuleau-Malot et al., 2014), the fact that we have subtracted a random quantity $\hat{\mathbf{C}}_{0}$ to $\mathbf{C}$ makes the approximation not valid for the uncentered quantity $\mathbf{C}$, as illustrated below. We cannot go back and forth by using the scaling and shifting properties of the Gaussian distributions. This is what we call the centering issue, problem which is actually completely related to the plug-in problem mentioned in (Gütig et al., 2001; Tuleau-Malot et al., 2014). Indeed, looking informally at (4), and doing as if $\hat{\mathbf{C}}_{0}$ was deterministic as for 

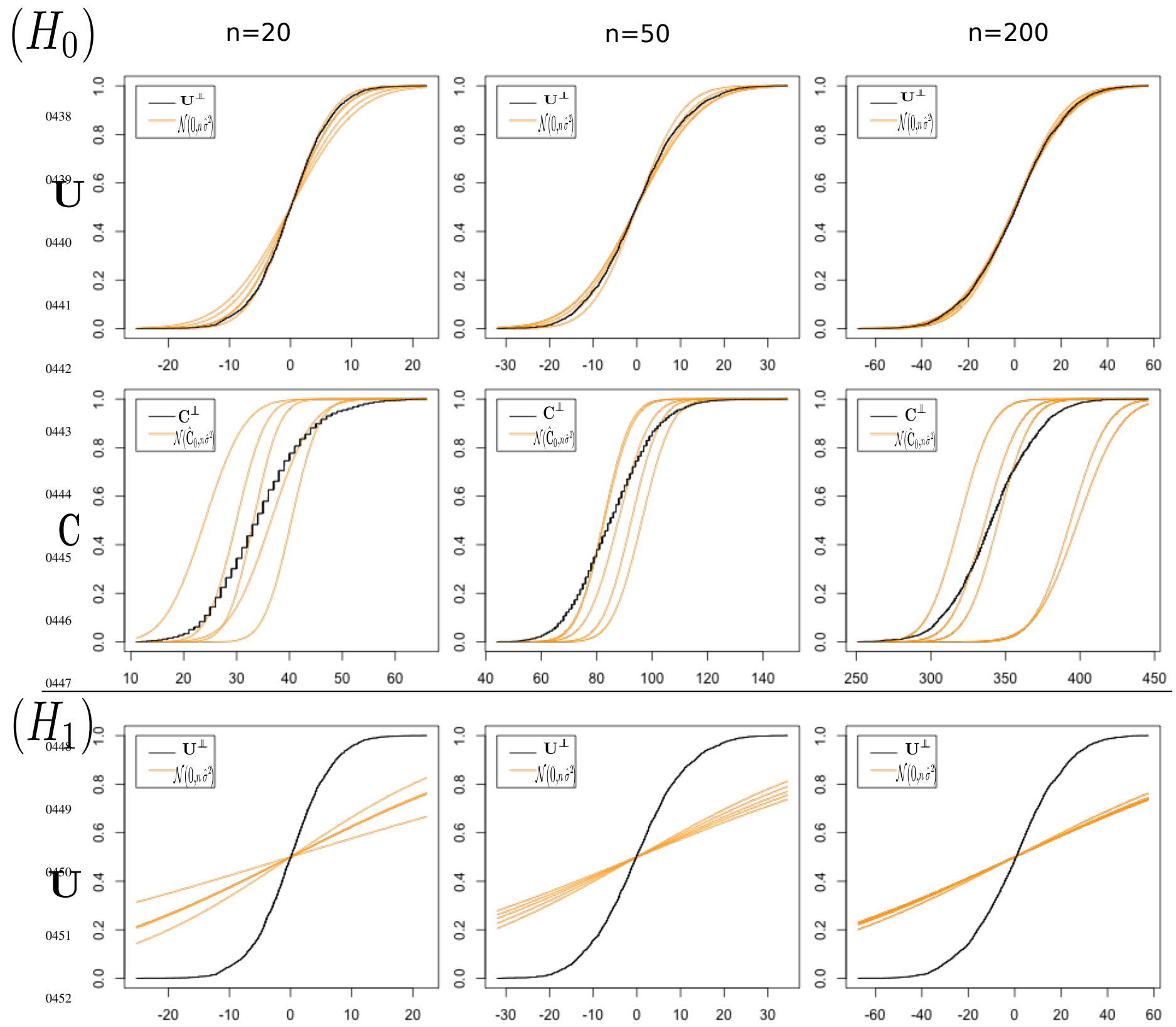

0453

Figure 3: Other Gaussian distribution approximations. Two first lines: c.d.f. of $\mathbf{U}$ and

0454

0455

0456

0457

0458

0459

$\mathrm{C}$ under $\left(H_{0}\right)$, obtained as in Figure 2. These c.d.f. are respectively compared with the

Gaussian c.d.f. with mean 0 and standard deviation $\sqrt{n} \hat{\sigma}\left(\mathbb{X}_{n}\right)$, and the Gaussian c.d.f. with mean $\hat{\mathbf{C}}_{0}\left(\mathbb{X}_{n}\right)$ and standard deviation $\sqrt{n} \hat{\sigma}\left(\mathbb{X}_{n}\right)$, for five different simulations of $\mathbb{X}_{n}$ under $\left(H_{0}\right)$. Third line: c.d.f. of $\mathbf{U}$ under $\left(H_{0}\right)$ computed as above, compared with the centered Gaussian c.d.f. with standard deviation $\sqrt{n} \hat{\sigma}\left(\mathbb{X}_{n}\right)$, for five different simulations of $\mathbb{X}_{n}$ under $\left(H_{1}\right)$ (same marginals as in the first two lines but $X^{1}=X^{2}$ ). 
with the observed sample. This does not change anything under $\left(H_{0}\right)$ since $\mathbb{X}_{n}$ is in this case distributed as $\mathbb{X}_{n}^{\Perp}$. Yet this is a particularly important sticking point if $\left(H_{0}\right)$ is not satisfied as one can see on the third line of Figure 3: the distribution of $\mathbf{U}\left(\mathbb{X}_{n}^{\Perp}\right)$ does not look like a centered Gaussian distribution of variance $n \hat{\sigma}^{2}\left(\mathbb{X}_{n}\right)$, when $\mathbb{X}_{n}$ does not satisfy $\left(H_{0}\right)$.

More importantly for the centering issue,the second line of Figure 3 shows that the approximation formulated in (6) is in fact misleading. To understand why, one needs to take into account the two following points.

(i) $\hat{\mathbf{C}}_{0}\left(\mathbb{X}_{n}^{\Perp}\right)$ moves around its expectation $c_{0}$ (which is also the expectation of $\mathbf{C}\left(\mathbb{X}_{n}^{\Perp}\right)$ ) with realizations of $\mathbb{X}_{n}^{\Perp}$. These fluctuations have an order of magnitude of $\sqrt{n}$ and are therefore perfectly observable on the distribution of $\mathbf{C}\left(\mathbb{X}_{n}^{\Perp}\right)$ whose variance is also of order $\sqrt{n}$.

(ii) $n \hat{\sigma}^{2}\left(\mathbb{X}_{n}^{\Perp}\right)$ estimates the variance of $\mathbf{U}\left(\mathbb{X}_{n}^{\Perp}\right)$ and not the one of $\mathbf{C}\left(\mathbb{X}_{n}^{\Perp}\right)$ or $\hat{\mathbf{C}}_{0}\left(\mathbb{X}_{n}^{\Perp}\right)$. This explains why not only the mean but also the variance are badly estimated in the second line of Figure 3. Two distinct kinds of randomness (the one coming from $\mathbf{C}\left(\mathbb{X}_{n}^{\Perp}\right)$ and the one coming from $\hat{\mathbf{C}}_{0}\left(\mathbb{X}_{n}^{\Perp}\right)$ ) have to be taken into account to estimate the variance of $\mathbf{U}\left(\mathbb{X}_{n}^{\Perp}\right)$.

As a conclusion of this first naive approach, the test of purely asymptotic nature, which consists in rejecting $\left(H_{0}\right)$ when $\mathbf{Z}^{\text {obs }}>z_{1-\alpha}$ may work for $n$ large enough, as the variance is here computed by considering the correctly recentered quantity $\mathbf{U}$, and this even if the behavior of $\mathbf{U}$ under $\left(H_{1}\right)$ is not good. However, an ad hoc and more naive test, based on an estimation of the variance of non recentered quantity $\mathbf{C}$ directly and without taking into account the fact that the centering term $\hat{\mathbf{C}}_{0}\left(\mathbb{X}_{n}\right)$ is also 
random, would not lead to a meaningful test with correct $p$-values: this is therefore a first example of centering issue.

\subsection{The bootstrap approaches}

In statistics, it is well known (Giné, 1997) that tests of purely asymptotic nature as the one presented above are less accurate for small values of $n$ than more involved procedures. In this article, the focus is on bootstrap/resampling procedures that are usually known to improve the performance from moderate to large sample sizes. Three main procedures are investigated that are all three based on a shuffling of the trials: the trial-shuffling introduced in (Pipa \& Grün, 2003; Pipa et al., 2003), the full bootstrap of independence and the permutation approach, the last two being more classical in statistics (see e.g. (Romano, 1989)), but also already used on spike train data (see e.g. (Ventura, 2010)).

The main common paradigm of these three methods, as described in the sequel, is that starting from an observation of the sample $\mathbb{X}_{n}$, they randomly generate, via a computer, another sample $\tilde{\mathbb{X}}_{n}$, whose distribution should be close to the distribution of $\mathbb{X}_{n}^{\Perp}$ (see also Figure 4$)$. 


\section{Trial-shuffling}

$$
\tilde{\mathbb{X}}_{n}=\mathbb{X}_{n}^{T S}=\left(\left(X_{i^{T S}(1)}^{1}, X_{j^{T S}(1)}^{2}\right), \ldots,\left(X_{i^{T S}(n)}^{1}, X_{j^{T S}(n)}^{2}\right)\right)
$$

where the $\left(i^{T S}(k), j^{T S}(k)\right)$ 's are $n$ i.i.d. couples drawn uniformly at random in $\{(i, j) / i=1, \ldots, n, j=1, \ldots, n, i \neq j\}$

In particular, the corresponding bootstrapped coincidence count is

$$
\mathbf{C}^{T S}=\mathbf{C}\left(\mathbb{X}_{n}^{T S}\right):=\sum_{k=1}^{n} \varphi\left(X_{i^{T S}(k)}^{1}, X_{j^{T S}(k)}^{2}\right)
$$

This algorithm seems natural with respect to (2) because it avoids the diagonal terms of the square $\{(i, j) / i=1, \ldots, n, j=1, \ldots, n\}$. Hence as a result,

$$
\mathbb{E}\left[\mathbf{C}^{T S}\right]=c_{0}=\mathbb{E}_{\Perp}[\mathbf{C}]
$$

Classical full bootstrap
\[ \tilde{\mathbb{X}}_{n}=\mathbb{X}_{n}^{*}=\left(\left(X_{i^{*}(1)}^{1}, X_{j^{*}(1)}^{2}\right), \ldots,\left(X_{i^{*}(n)}^{1}, X_{j^{*}(n)}^{2}\right)\right) \]
where the $n$ couples $\left(i^{*}(k), j^{*}(k)\right)$ are i.i.d. and where $i^{*}(k)$ and $j^{*}(k)$ are drawn
uniformly and independently at random in $\{1, \ldots, n\}$.
In particular, the corresponding bootstrapped coincidence count is
\[ \mathbf{C}^{*}=\mathbf{C}\left(\mathbb{X}_{n}^{*}\right):=\sum_{k=1}^{n} \varphi\left(X_{i^{*}(k)}^{1}, X_{j^{*}(k)}^{2}\right) . \]

Note that this algorithm draws uniformly at random in the square $\{(i, j) / i=$ $1, \ldots, n, j=1, \ldots, n\}$ and therefore does not avoid the diagonal terms. The idea behind this algorithm is to mimic the independence under $\left(H_{0}\right)$ of $X_{k}^{1}$ and $X_{k}^{2}$ by drawing the 
indexes $i^{*}(k)$ and $j^{*}(k)$ independently. However

$$
\mathbb{E}\left[\mathbf{C}^{*}\right]=n\left(\frac{1}{n} \mathbb{E}\left[\varphi\left(X^{1}, X^{2}\right)\right]+\frac{n-1}{n} \mathbb{E}_{\Perp}\left[\varphi\left(X^{1, \Perp}, X^{2, \Perp}\right)\right]\right) .
$$

Hence under $\left(H_{0}\right), \mathbb{E}_{\Perp}\left[\mathbf{C}^{*}\right]=c_{0}$ but, under $\left(H_{1}\right), \mathbb{E}\left[\mathbf{C}^{*}\right]$ and $c_{0}$ are only asymptotically equivalent.

Permutation
\[ \tilde{\mathbb{X}}_{n}=\mathbb{X}_{n}^{\Pi_{n}}=\left(\left(X_{1}^{1}, X_{\Pi_{n}(1)}^{2}\right), \ldots,\left(X_{n}^{1}, X_{\Pi_{n}(n)}^{2}\right)\right) \]
where $\Pi_{n}$ is a permutation drawn uniformly at random in the group of permutations
$\mathfrak{S}_{n}$ of the set of indexes $\{1, \ldots, n\}$.
In particular, the corresponding bootstrapped coincidence count is
\[ \mathbf{C}^{\star}=\mathbf{C}\left(\mathbb{X}_{n}^{\Pi_{n}}\right):=\sum_{i=1}^{n} \varphi\left(X_{i}^{1}, X_{\Pi_{n}(i)}^{2}\right) . \]

The idea is to use permutations to avoid picking twice the same spike train of the same trial. In particular under $\left(H_{0}\right)$, the sum in $\mathbf{C}^{\star}$ is still a sum of independent variables, which is not the case in both of the previous algorithms. However, under $\left(H_{1}\right)$, the behavior is not as limpid. As for the full bootstrap,

$$
\mathbb{E}\left[\mathbf{C}^{\star}\right]=n\left(\frac{1}{n} \mathbb{E}\left[\varphi\left(X^{1}, X^{2}\right)\right]+\frac{n-1}{n} \mathbb{E}_{\Perp}\left[\varphi\left(X^{1}, X^{2}\right)\right]\right) .
$$

Hence under $\left(H_{1}\right), \mathbb{E}\left[\mathbf{C}^{\star}\right]$ and $c_{0}$ are also only asymptotically equivalent.

To compare those three bootstrap/resampling algorithms, the first thing to wonder is whether, at least under $\left(H_{0}\right)$, the introduced extra randomness has not impacted the distribution. More precisely, as stated above, all three procedures satisfy

$$
\mathbb{E}_{\Perp}\left[\mathbf{C}\left(\tilde{\mathbb{X}}_{n}\right)\right]=\mathbb{E}_{\Perp}\left[\mathbf{C}\left(\mathbb{X}_{n}^{\Perp}\right)\right]=c_{0},
$$


but is the full unconditional distribution of $\mathbf{C}\left(\tilde{\mathbb{X}}_{n}\right)$ the same as the one of $\mathbf{C}\left(\mathbb{X}_{n}^{\Perp}\right)$ ? See Figure 4 for a more visual explanation of what is the unconditional distribution.

The first line of Figure 5 shows as expected that the permutation does not change the distribution of $\mathbb{X}_{n}^{\Perp}$, since, as said above, no spike train is picked twice. However, clearly the trial-shuffling and the full bootstrap have not the same property, even if the distributions are quite close.

Nevertheless, this is not completely convincing. Indeed and as already mentioned in Figure 4, the main particularity of surrogate data procedures is to be able for one current observation of $\mathbb{X}_{n}$ to generate several surrogate data sets, that is several realizations of $\tilde{\mathbb{X}}_{n}$, and to obtain not the unconditional distribution of $\mathbf{C}\left(\tilde{\mathbb{X}}_{n}\right)$ but the conditional distribution of $\mathbf{C}\left(\tilde{\mathbb{X}}_{n}\right)$ given $\mathbb{X}_{n}$. What is important to emphasize is that this conditional distribution (which is the one to which one has access in practice) actually depends on the original data set. This is why on the second line of Figure 5, are given five realizations of the conditional cumulative distribution function: since this is a simulation, we are able to produce 5 "original" data sets and to see how the conditional distribution fluctuates thanks to the Nature randomness as described in Figure 4. What we can expect is that as a proxy, this conditional distribution, which is the only accessible one, will be close to the one we would like to know, that is the distribution of $\mathbf{C}^{\Perp}$.

However none of the three conditional distributions seems to fit the distribution of $\mathbf{C}\left(\mathbb{X}_{n}^{\Perp}\right)$. One may eventually think that this is due to the Monte-Carlo approximation of the conditional distributions, but for the trial-shuffling approach, Pipa and Grün developed an algorithm for exact computation of the conditional distribution (Pipa et al., 2003): both Monte-Carlo and exact conditional distribution are so close that it is diffi- 
cult to make any difference between them.

Hence there should be another explanation. In fact, the curves on the second line of Figure 5 are similar to the ones on the second line of Figure 3. In both set-ups, one wonders if the distribution of $\mathbf{C}\left(\mathbb{X}_{n}^{\Perp}\right)$ can or cannot be approximated by a distribution depending on the observation of $\mathbb{X}_{n}$ : a very basic Gaussian distribution for Figure 3 and a more intricate distribution using the bootstrap paradigm for Figure 5. In both cases, the conditional c.d.f. are widely spread around the aim which is the distribution of $\mathrm{C}\left(\mathbb{X}_{n}^{\Perp}\right)$. Since the explanation for Figure 3 was a centering defect that can be corrected by considering $\mathbf{U}$, the explanation here is a centering defect for the procedures based on a shuffling of the trials too, and this can also be corrected as one can see below.

\subsection{Which centering for which bootstrap ?}

To understand the centering issue of the procedures based on a shuffling of the trials, one needs to understand more precisely the mathematical results on bootstrap.

The precursor work of Bickel and Freedman (Bickel \& Freedman, 1981) on the bootstrap of the mean can be heuristically explained as follows. Given a $n$ sample of i.i.d. real random variables $\mathbb{Y}_{n}=\left(Y_{1}, \ldots, Y_{n}\right)$ with mean $m$ and a corresponding bootstrap sample $\mathbb{Y}_{n}^{*}$, it is not possible to estimate the distribution of the empirical mean $\bar{Y}=(1 / n) \sum_{i=1}^{n} Y_{i}$ directly. However one can estimate the centered distribution, i.e. the distribution of $\bar{Y}-m=\bar{Y}-\mathbb{E}[\bar{Y}]$. To do so, it is sufficient to replace "empirical mean" by "empirical bootstrap mean" and "expectation" by "conditional expectation". More explicitly, denoting by $\bar{Y}^{*}$ the empirical mean of the bootstrap sample $\mathbb{Y}_{n}^{*}$, the distribution of $\bar{Y}-\mathbb{E}[\bar{Y}]$ is approximated by the conditional distribution given $\mathbb{Y}_{n}$ of 


$$
\bar{Y}^{*}-\mathbb{E}\left[\bar{Y}^{*} \mid \mathbb{Y}_{n}\right]
$$

More generally, the bootstrap approaches that have been proved to work from a mathematical point of view are all based on centered quantities (Giné, 1997): this is $\bar{Y}-m$ in the previous example but this can also be centered U-statistics. However, this cannot be $\mathbf{C}$, which is not centered, as one can see in Figure 5.

A suitable quantity in our context is $\mathbf{U}$ given in (3), since it has zero mean under $\left(H_{0}\right)$. Indeed, by the bootstrap paradigm recalled above, the distribution of $\mathbf{U}\left(\mathbb{X}_{n}\right)$ under $\left(H_{0}\right)$, that is of $\mathbf{U}\left(\mathbb{X}_{n}^{\Perp}\right)$ (which has zero mean), should be well approximated by the distribution of $\mathbf{U}\left(\tilde{\mathbb{X}}_{n}\right)-\mathbb{E}\left[\mathbf{U}\left(\tilde{\mathbb{X}}_{n}\right) \mid \mathbb{X}_{n}\right]$.

For the trial-shuffling, since

$$
\mathbf{U}\left(\mathbb{X}_{n}^{T S}\right)=\sum_{k=1}^{n} \varphi\left(X_{i^{T S}(k)}^{1}, X_{j^{T S}(k)}^{2}\right)-\frac{1}{n-1} \sum_{k \neq k^{\prime}} \varphi\left(X_{i^{T S}(k)}^{1}, X_{j^{T S}\left(k^{\prime}\right)}^{2}\right)
$$

one can easily see that because the couple $\left(i^{T S}(k), j^{T S}(k)\right)$ is drawn uniformly at random in the set of the $(i, j)$ 's such that $i \neq j$ (set of cardinality $n(n-1)$ ),

$$
\begin{aligned}
\mathbb{E}\left[\mathbf{U}\left(\mathbb{X}_{n}^{T S}\right) \mid \mathbb{X}_{n}\right] & =\frac{1}{n-1} \sum_{i \neq j} \varphi\left(X_{i}^{1}, X_{j}^{2}\right)-\frac{1}{n} \sum_{i, j} \varphi\left(X_{i}^{1}, X_{j}^{2}\right) \\
& =\frac{\hat{\mathbf{C}}_{0}\left(\mathbb{X}_{n}\right)-\mathbf{C}\left(\mathbb{X}_{n}\right)}{n} \\
& =-\frac{\mathbf{U}\left(\mathbb{X}_{n}\right)}{n}
\end{aligned}
$$

Hence the quantity that needs to be computed on the surrogate data set when applying the trial-shuffling method is

$$
\tilde{\mathbf{U}}^{T S}=\tilde{\mathbf{U}}\left(\mathbb{X}_{n}^{T S}\right)=\mathbf{U}\left(\mathbb{X}_{n}^{T S}\right)+\frac{\mathbf{U}\left(\mathbb{X}_{n}\right)}{n}
$$

Furthermore, similar computations show that the full bootstrap and the permutation satisfy

$$
\mathbb{E}\left[\mathbf{U}\left(\mathbb{X}_{n}^{*}\right) \mid \mathbb{X}_{n}\right]=\mathbb{E}\left[\mathbf{U}\left(\mathbb{X}_{n}^{\Pi_{n}}\right) \mid \mathbb{X}_{n}\right]=0
$$


Hence, $\mathbf{U}\left(\mathbb{X}_{n}^{*}\right)$ and $\mathbf{U}\left(\mathbb{X}_{n}^{\Pi_{n}}\right)$ can be computed directly on the surrogate data sets when applying either the Full Bootstrap or the Permutation methods.

Figure 6 shows the quality of approximation of the distribution of $\mathbf{U}\left(\mathbb{X}_{n}^{\Perp}\right)$ by the conditional distribution given the observation of either $\tilde{\mathbf{U}}^{T S}, \mathbf{U}^{T S}=\mathbf{U}\left(\mathbb{X}_{n}^{T S}\right), \mathbf{U}^{*}=$ $\mathbf{U}\left(\mathbb{X}_{n}^{*}\right)$ or $\mathbf{U}^{\star}=\mathbf{U}\left(\mathbb{X}_{n}^{\Pi_{n}}\right)$. Contrary to Figure 5 , the conditional distributions of $\mathbf{U}^{*}$ and $\mathrm{U}^{\star}$ do not spread widely around the target distribution but are accurate approximations not only under $\left(H_{0}\right)$ but even if the observed sample is simulated under $\left(H_{1}\right)$, which is in complete accordance with the mathematical results of consistence proven in (Albert et al., 2015). The approximation is just as accurate when using the recentered quantity $\tilde{\mathbf{U}}^{T S}$ to mimic the distribution of $\mathbf{U}^{\Perp}$ but it is not for simply $\mathbf{U}^{T S}$, the difference between the conditional c.d.f. of $\tilde{\mathbf{U}}^{T S}$ and the one of $\mathbf{U}^{T S}$ being particularly visible under $\left(H_{1}\right)$ when $X^{1}=X^{2}$. This means that one definitely need to recenter the quantities that are computed on the surrogate data set by subtracting their conditional expectation given the original data set to obtain a correct fit of the desired centered distribution under independence.

Hence, as explained by the computations above, in a trial-shuffling approach, the correctly recentered version leads to the correct bootstrap distribution. Note finally that this corroborates the previous intuition: the reason why the approximation works for $\mathbf{U}$ and not for $\mathbf{C}$ is a centering issue, that is exactly the same as for the first approach of Figure 3. The centering is indeed random as in Figure 3 (here it can be viewed as $\left.\mathbb{E}\left[\mathbf{C}\left(\tilde{\mathbb{X}}_{n}\right) \mid \mathbb{X}_{n}\right]\right)$ and one needs to take it into account to have a correct approximation.

Finally an extra simplification holds in the permutation case, which may seem very surprising. 
One can easily rewrite on the one hand,

$$
\mathbf{U}\left(\mathbb{X}_{n}\right)=\left(1+\frac{1}{n-1}\right) \mathbf{C}\left(\mathbb{X}_{n}\right)-\frac{1}{n-1} \sum_{i, j} \varphi\left(X_{i}^{1}, X_{j}^{2}\right)
$$

and, on the other hand, for the permutation sample

$$
\mathbf{U}\left(\mathbb{X}_{n}^{\Pi_{n}}\right)=\left(1+\frac{1}{n-1}\right) \mathbf{C}\left(\mathbb{X}_{n}^{\Pi_{n}}\right)-\frac{1}{n-1} \sum_{i, j} \varphi\left(X_{i}^{1}, X_{j}^{2}\right)
$$

Note that the sum $\sum_{i, j} \varphi\left(X_{i}^{1}, X_{j}^{2}\right)$ is invariant by the action of the permutation. Hence if $u_{t}^{\star}$ denotes the quantile of order $t$ of the conditional distribution of $\mathbf{U}\left(\mathbb{X}_{n}^{\Pi_{n}}\right)$ given $\mathbb{X}_{n}$ and if $c_{t}^{\star}$ denotes the quantile of order $t$ of the conditional distribution of $\mathbf{C}\left(\mathbb{X}_{n}^{\Pi_{n}}\right)$ given $\mathbb{X}_{n}$, this very simple relationship holds

$$
u_{t}^{\star}=\left(1+\frac{1}{n-1}\right) c_{t}^{\star}-\frac{1}{n-1} \sum_{i, j} \varphi\left(X_{i}^{1}, X_{j}^{2}\right) .
$$

Hence the test that rejects $\left(H_{0}\right)$ when $\mathbf{U}\left(\mathbb{X}_{n}\right)>u_{1-\alpha}^{\star}$ is exactly the one that rejects $\left(H_{0}\right)$ when $\mathbf{C}\left(\mathbb{X}_{n}\right)>c_{1-\alpha}^{\star}$. Therefore despite the fact that the conditional distribution of $\mathbf{C}\left(\mathbb{X}_{n}^{\Pi_{n}}\right)$ is not close at all to the one of $\mathbf{C}\left(\mathbb{X}_{n}^{\Perp}\right)$, the test based on $\mathbf{C}$ works, because it is equivalent to the test based on $\mathbf{U}$, for which the approximation of the conditional distribution works. Note however that this phenomenon happens only in the permutation approach, but not in the trial-shuffling or the full bootstrap approaches.

\subsection{Practical testing procedures and $p$-values}

From the considerations given above, five different tests may be investigated, the first one based on a purely asymptotic approach, and the four other ones based on resampling approaches, with critical values approximated through a Monte-Carlo method. For each test, the corresponding $p$-values (i.e. the values of $\alpha$ for which the test passes from acceptance to rejection) are given. 
The naive test $\mathbf{( N )}$. It consists in rejecting $\left(H_{0}\right)$ when

$$
\mathbf{Z}^{\text {obs }} \geq z_{1-\alpha}
$$

The corresponding $p$-value is given by:

$$
1-\Phi\left(\mathbf{Z}^{o b s}\right)
$$

where $\Phi$ is the c.d.f. of a standard Gaussian distribution.

The Trial-Shuffling test, version C (TSC). It consists in rejecting $\left(H_{0}\right)$ when

$$
\mathbf{C}^{o b s} \geq \hat{c}_{1-\alpha}^{T S}
$$

where $\hat{c}_{1-\alpha}^{T S}$ is the empirical quantile of order $(1-\alpha)$ of the conditional distribution of $\mathbf{C}^{T S}$ given $\mathbb{X}_{n}$. This empirical quantile is estimated over $B$ ( $B=10000$ usually) realizations $\mathbf{C}_{1}^{T S}, \ldots, \mathbf{C}_{B}^{T S}$ given the observed sample $\mathbb{X}_{n}$. The corresponding $p$-value is given by:

$$
\frac{1}{B} \sum_{i=1}^{B} \mathbf{1}_{\mathbf{C}_{i}^{T S} \geq \mathbf{C}^{\text {obs }}} .
$$

Despite the centering defect of this method underlined in Section 3.3, we kept this test in the present study since it corresponds to the one programmed in (Pipa \& Grün, 2003) and since it is widely applied in the neuroscience literature.

The Trial-Shuffling test, version recentered U (TSU). It consists in rejecting $\left(H_{0}\right)$ when

$$
\mathbf{U}^{o b s} \geq \hat{w}_{1-\alpha}^{T S}
$$

where $\hat{w}_{1-\alpha}^{T S}$ is the empirical quantile of order $(1-\alpha)$ of the conditional distribution of $\tilde{\mathbf{U}}^{T S}$ (the correctly recentered quantity) given $\mathbb{X}_{n}$. This empirical quantile and the 
corresponding $p$-value are obtained in away similar to the above (TSC), based on $B$ realizations $\tilde{\mathbf{U}}_{1}^{T S}, \ldots, \tilde{\mathbf{U}}_{B}^{T S}$ of $\tilde{\mathbf{U}}\left(\mathbb{X}_{n}^{T S}\right)$ given $\mathbb{X}_{n}$.

The Full Bootstrap test, version U (FBU). It consists in rejecting $\left(H_{0}\right)$ when

$$
\mathbf{U}^{o b s} \geq \hat{u}_{1-\alpha}^{*},
$$

where $\hat{u}_{1-\alpha}^{*}$ is the empirical quantile of order $(1-\alpha)$ of the conditional distribution of $\mathbf{U}^{*}$ given $\mathbb{X}_{n}$. This empirical quantile and the corresponding $p$-value are obtained in a way similar to the above (TSC), based on $B$ realizations $\mathbf{U}_{1}^{*}, \ldots, \mathbf{U}_{B}^{*}$ of $\mathbf{U}\left(\mathbb{X}_{n}^{*}\right)$ given $\mathbb{X}_{n}$

The permutation test $(\mathbf{P})$. The reader may think that it should consist in rejecting $\left(H_{0}\right)$ when

$$
\mathbf{C}^{o b s} \geq \hat{c}_{1-\alpha}^{\star}
$$

where $\hat{c}_{1-\alpha}^{\star}$ is the empirical quantile of order $(1-\alpha)$ of the conditional distribution of $\mathrm{C}^{\star}$ given $\mathbb{X}_{n}$. Yet the test by permutation is in fact directly defined by its $p$-value, which is slightly different here, equal to:

$$
\frac{1}{B+1}\left(1+\sum_{i=1}^{B} \mathbf{1}_{\mathbf{C}_{i}^{*} \geq \mathbf{C}^{o b s}}\right)
$$

where $\mathbf{C}_{1}^{\star}, \ldots, \mathbf{C}_{B}^{\star}$ are $B$ realizations of $\mathbf{C}\left(\mathbb{X}_{n}^{\Pi_{n}}\right)$ given $\mathbb{X}_{n}$. The permutation test then consists in rejecting $\left(H_{0}\right)$ when this $p$-value is less than $\alpha$. Indeed, such a permutation test, with such a slightly different version of $p$-value, has been proved to be exactly of level $\alpha$, whatever $B$ (Romano \& Wolf, 2005), thanks to exchangeability properties of random permutations. 
Note however that such a slight correction does not work for full bootstrap or trial-shuffling approaches, where the tests are only guaranteed to be asymptotically of level $\alpha$.

Saying that a test rejects at level $\alpha$ (or that its False Positive (FP) rate is smaller than $\alpha$ ) is exactly equivalent to saying that its $p$-value is less than $\alpha$. If a test is of level $\alpha$ for any $\alpha$ in $(0,1)$, the c.d.f. of its $p$-values should therefore be smaller than the one of a uniform variable (i.e. the diagonal) under $\left(H_{0}\right)$. Between several tests with this guarantee, the less conservative one is the one for which the c.d.f of its $p$-values is the closest to the diagonal. The left hand-side of Figure 7 shows the c.d.f. under $\left(H_{0}\right)$ of the corresponding $p$-values for the five considered testing procedures and focuses on small $p$-values, which are the only ones usually involved in testing, to highlight the main differences between the five methods. For the chosen small value of $n(n=20)$, the c.d.f. of the (TSU) and (FBU) $p$-values are almost identical and above the diagonal, meaning that the corresponding tests do not guarantee the level. On the contrary, the c.d.f. of the (N) and (TSC) p-values are clearly under the diagonal and far from it, meaning that the corresponding tests are too conservative. As guaranteed by (Romano $\&$ Wolf , 2005), the permutation approach guarantees the level of the test: the c.d.f. of the $(\mathrm{P}) p$-values is also under the diagonal, under $\left(H_{0}\right)$, but much closer to the diagonal than the one of the (N) and (TSC) $p$-values.

Furthermore, the behavior of the c.d.f. of the $p$-values under $\left(H_{1}\right)$ gives an indication of the power of the test. Indeed this c.d.f associates to each $\alpha$ in $(0,1)$, the (estimated) probability that the test rejecting independence when its $p$-value is less than $\alpha$, actually rejects independence. This probability is, under $\left(H_{1}\right), 1$ minus the False 
Negative $(\mathrm{FN})$ rate. It can also be seen as the power of the test. Hence among the tests that guarantee the level, the permutation test $(\mathrm{P})$ is the one with the smallest FN rate, that is the most powerful one.

Note that other simulations in more various cases have been performed in (Albert et al., 2015) leading to the same conclusion.

We have also performed some simulations for which the firing rate is not constant across the trials. The results are displayed on Figure 8. It is important to note that the independence is rejected (that is when the $p$-values are clearly small, with a c.d.f. clearly above the diagonal) only when the rates of each components $X^{1}$ and $X^{2}$ progress in a coordinate way (see Figure 8.A). If only one rate varies (see Figure 8.B), the $p$-values are all close to diagonal except for (TSC), for which the distribution approximation does not work as we showed above. The same appears in the set-up considered by (Grün et al, 2003; Grün, 2009) (see Figure 8.C) with $p$-values even closer to the diagonal, because the number of trials is larger. Note that this set-up was given in (Grün, 2009) as the worst case scenario of non-stationarity across trials for the trial-shuffling method and it was stated that this is due to a violation of the underlying assumption of nonstationarity across trials. However, as shown by Figure 8 in those two last situations, the $p$-values behave as under $\left(H_{0}\right)$, except maybe for (TSC), and we believe that this is explained not by a violation of the i.i.d. assumption on the trials but by a centering defect, as explained above. As announced in the introduction, cross-trials stationarity is not equivalent to the i.i.d. assumptions on the trials and this explains also why the correctly centered bootstrap methods work in this non-stationary case. We discuss in more detail this behavior in the discussion (see Section 5). 
In the sequel, since the permutation method is the only one able to guarantee the level of the test (that is to control the (FP) rate) even for a very small number of observation (see Figure 7), we focus on the permutation approach, keeping also the trial-shuffling version $\mathbf{C}$ approach, denoted by (TSC) on the graphs, as a variant of the method developed in (Pipa \& Grün, 2003).

\section{Permutation UE}

\subsection{Description of the complete multiple testing algorithm}

To detect precise locations of dependence periods that can be matched to some experimental or behavioral events, the third step (point (iii) of the introduction) of a UE method is classically to consider a family of windows $\mathcal{W}$ of cardinal $K$, which is a collection of potentially overlapping intervals $[a, b]$ covering the whole interval $[0, T]$ on which trials have been recorded (Grün et al., 1999; Tuleau-Malot et al., 2014). Then, some independence tests are implemented on each window of the collection. Here we propose a complete algorithm which takes into account the multiplicity of the tests, and which moreover enables to see if the coincidence count is significantly too large or too small on each window as in (Tuleau-Malot et al., 2014). 


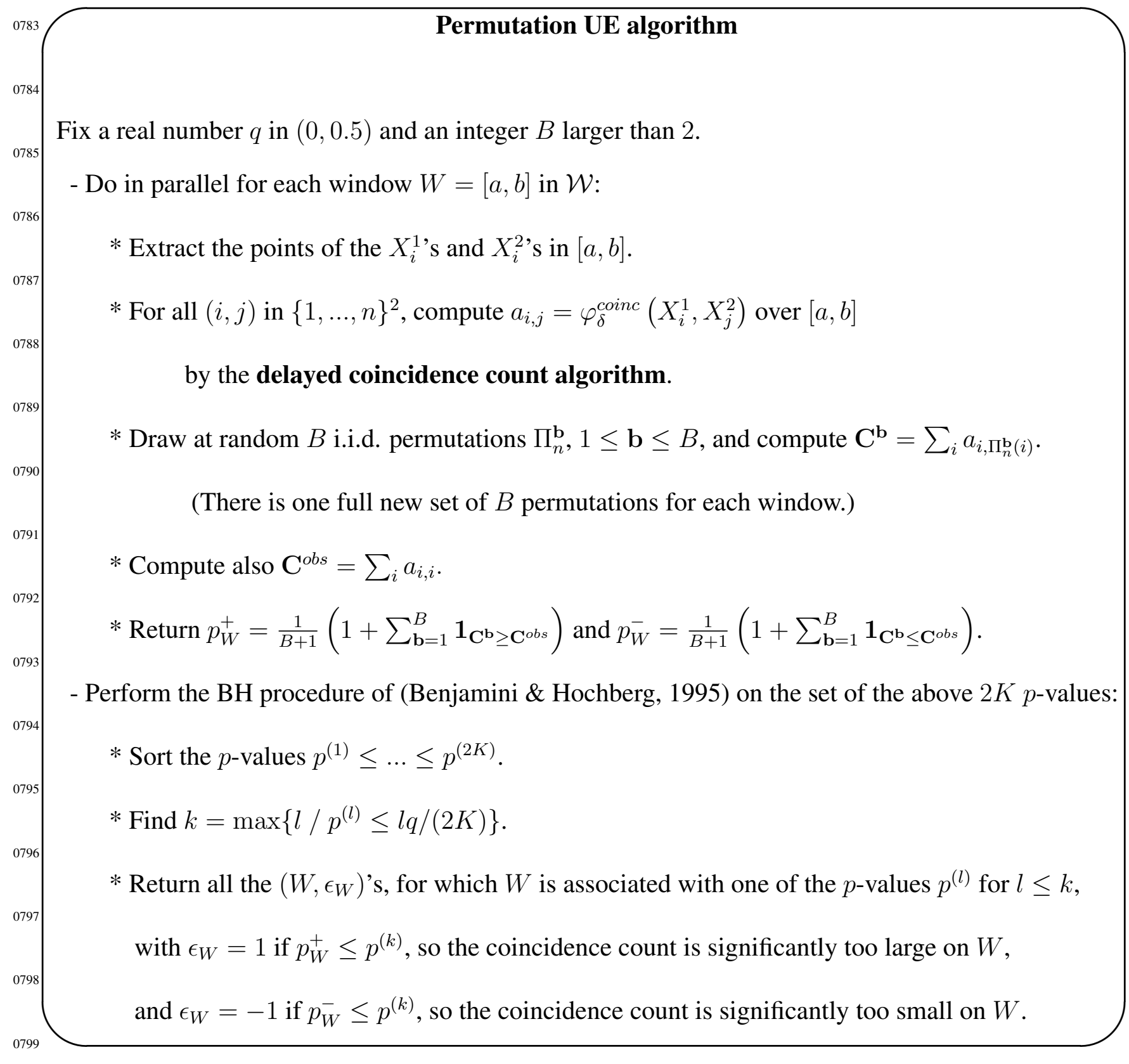

The code has been parallelized in $\mathrm{C}++$ and interfaced with $\mathrm{R}$. The full corresponding R-package is still a work in progress but actual codes are available at https://github.com/ybouret/neuro-stat. 
Malot et al., 2014), but adapted to non necessarily symmetric distributions ${ }^{2}$. In several applications, neuroscientists are interested in detecting dependence periods for which the coincidence count is only significantly too large. In this case, one can use the restricted set of the $p_{W}^{+}$'s.

From a mathematical point of view, if the considered windows are disjoint and if the spike trains are Poisson processes that are non necessarily stationary, the False Discovery Rate (FDR) ${ }^{3}$ of the above multiple testing procedure can be mathematically proven $^{4}$ to be controlled by $q$ for any $B \geq 2$. The problem of mathematically proving that $\mathrm{BH}$ procedure guarantees an FDR smaller than $q$ without those restrictions is very difficult even in simple situations such as the Gaussian regression framework (Benjamini \& Yekutieli, 2001), while it is usually observed in practice that the FDR is still controlled by $q$. However it has been proved in (Benjamini \& Yekutieli, 2001) that for any framework and therefore in particular for the most general setting of Permutation $\mathrm{UE}$, the FDR of $\mathrm{BH}$ is always smaller than $q \sum_{\ell=1}^{2 K} \ell^{-1} \simeq q \ln (2 K)$. Hence, for instance, with 50 windows and $q=0.01$, we are still mathematically guaranteeing that the FDR of Permutation UE described in the above sidebar is whatever the underlying distribution controlled by 0.052 . Moreover the distributions that are reaching this rate are so particular that it is often advised even by mathematical experts of multiple testing

\footnotetext{
${ }^{2}$ Note in particular that for a fixed $W$, one cannot have both $p_{W}^{+}<0.5$ and $p_{W}^{-}<0.5$ and therefore, if a $W$ is detected, it can only be because of one of the two situations, $p_{W}^{+} \leq p^{(k)}$ or $p_{W}^{-} \leq p^{(k)}$, which cannot happen simultaneously.

3 see (Tuleau-Malot et al., 2014) or Table 1 for a precise definition

${ }^{4}$ The $p_{W}^{+}$'s are independent random variables such that $\mathbb{P}_{\Perp}\left(p_{W}^{+} \leq \alpha\right) \leq \alpha$ for all $\alpha$ in $[0,1]$ (Benjamini \& Yekutieli, 2001; Romano \& Wolf , 2005).
} 
to do as if the control of the FDR by $q$ holds as soon as typical simulations do not show otherwise.

\subsection{Comparison on simulations}

Two sets of simulations have been carried out. The first one, namely Experiment 1, combines different point processes encountered in the literature (homogeneous, and inhomogeneous Poisson processes, Hawkes processes), and different kinds of dependences. It is described in Figure 9.A. The second one, namely Experiment 2, consists of simple independent homogeneous Poisson processes on the whole interval $[0,2]$, as described in Table 1. The corresponding results are described in Table 1 and one run of simulation of the Permutation UE method is presented in Figure 9. Four methods have been compared:

- the MTGAUE method of Tuleau-Malot et al. (2014) which assumes both processes to be homogeneous Poisson processes, with $q=0.05$,

- the Trial-Shuffling, version C (TSC) which corresponds to the method of Pipa \& Grün (2003), which has been programmed with the delayed coincidence count described above and which has not been corrected for multiplicity, that is with level $\alpha=0.05$ on all windows,

- the same as above but corrected by Benjamini and Hochberg procedure (TSC + $\mathrm{BH})$, that is with $q=0.05$,

- the Permutation UE approach described above, with $q=0.05$. 
In Figure 9.B, several $\delta$, that is several delays for the delayed coincidence counts, have been tested and each line corresponds to a different value of $\delta$. We see that except for very few false positives, the method is able to detect the correct dependence features and that it is also able to distinguish between situations where there are too many coincidences (bands delimited by plain black lines and containing red crosses) or too few coincidences (bands delimited by dotted black lines and containing blue crosses), the bands being what should be detected and the crosses being what is indeed detected in the simulation. Moreover one sees that even if there are some variations, detections occur for all reasonable values of $\delta$.

The permutation approach always guarantees an FDR less than the prescribed level of 0.05 whereas MTGAUE does not when the homogeneous Poisson assumption fails (Experiment 1). The classical trial-shuffling method (where dependence detection occurs each time the $p$-value is less than 0.05 ) seems to have comparable results in terms of both FDR and False Non Discovery Rate (FNDR) on Experiment 1 but fails to control the FDR on the most basic situation, namely purely independent processes (Experiment 2). Adding a Benjamini-Hochberg step of selection of $p$-values to the trialshuffling makes it more robust but at the price of a much larger FNDR with respect to the Permutation UE method, a fact which is consistent with the conservativeness shown in Figure 7.

\subsection{Comparison on real data}

Behavioral procedure. The data used in this theoretical article to test the dependence detection ability of the four methods were already partially published in previous ex- 


\begin{tabular}{l|c|c|c|c|}
\multicolumn{1}{c|}{0875} & & Independ. & Depend. & Total \\
\cline { 2 - 5 } 0876 & & $S$ & $R$ \\
\hline \multirow{4}{*}{0877} & Rejected & $V$ & $T$ & $m-R$ \\
\cline { 2 - 5 } 0878 & Accepted & $U$ & $m-m_{0}$ & $m$ \\
\cline { 2 - 6 } & Total & $m_{0}$ & $m$ &
\end{tabular}

0880 to the Permutation UE method ( $B=10000, q=0.05)$.

\begin{tabular}{|c|c|c|c|c|}
\hline & \multicolumn{2}{|c|}{ Experiment 1 } & \multicolumn{2}{c|}{ Experiment 2 } \\
& FDR & FNDR & FDR & FNDR \\
\hline MTGAUE & 0.10 & 0.17 & 0.04 & 0 \\
\hline TSC & 0.01 & 0.26 & 0.25 & 0 \\
\hline TSC + BH & 0 & 0.32 & 0 & 0 \\
\hline P & 0.01 & 0.23 & 0.02 & 0 \\
\hline
\end{tabular}

Table 1: False Discovery and Non Discovery Rates. On the left hand-side, the classical table for multiple testing adapted to our dependence framework, with a total number of tests $m=2 K$. On the right hand-side, estimated FDR and FNDR over 1000 runs, FDR being defined by $\mathbb{E}\left[(V / R) \mathbf{1}_{R>0}\right]$ and FNDR being defined by $\mathbb{E}\left[(T /(m-R)) \mathbf{1}_{m-R>0}\right]$. Experiment 1 is described in Figure 9, Experiment 2 consists of two independent homogeneous Poisson processes of firing rate $60 \mathrm{~Hz}$ on $[0,2]$. The set of windows is as in Figure 9. There are 50 trials and $\delta=0.01 \mathrm{~s}$. MTGAUE is the method described in (Tuleau-Malot et al., 2014) with $q=0.05$. (TSC) is the trial-shuffling method with Monte-Carlo approximation $(B=10000)$ and the selected windows are the ones whose $p$-value are less than $0.05 .(\mathrm{TSC}+\mathrm{BH})$ is the same method, except that the multiplicity of the tests is corrected by a Benjamini-Hochberg procedure $(q=0.05)$. (P) corresponds

perimental studies (Riehle et al., 2000; Grammont \& Riehle, 2003; Riehle et al., 2006) and also used in (Tuleau-Malot et al., 2014). These data were collected on a 5-year-old male Rhesus monkey who was trained to perform a delayed multidirectional pointing task. The animal sat in a primate chair in front of a vertical panel on which seven touch-sensitive light-emitting diodes were mounted, one in the center and six placed 
equidistantly (60 degrees apart) on a circle around it. The monkey had to initiate a trial by touching and then holding with the left hand the central target. After a fix delay of $500 \mathrm{~ms}$, the preparatory signal (PS) was presented by illuminating one of the six peripheral targets in green. After a delay of either $600 \mathrm{~ms}$ or $1200 \mathrm{~ms}$, selected at random with various probability, it turned red, serving as the response signal and pointing target. During the first part of the delay, the probability $p_{\text {resp }}$ for the response signal to occur at $(500+600) \mathrm{ms}=1.1 \mathrm{~s}$ was 0.3. Once this moment passed without signal occurrence, the conditional probability for the signal to occur at $(500+600+600) \mathrm{ms}=1.7 \mathrm{~s}$ changed to 1 . The monkey was rewarded by a drop of juice after each correct trial. Reaction time (RT) was defined as the release of the central target. Movement time (MT) was defined as the touching of the correct peripheral target.

Recording technique. Signals recorded from up to seven microelectrodes (quartz insulated platinum-tungsten electrodes, impedance: $2-5 \mathrm{M} \Omega$ at $1000 \mathrm{~Hz}$ ) were amplified and band-pass filtered from $300 \mathrm{~Hz}$ to $10 \mathrm{kHz}$. Using a window discriminator, spikes from only one single neuron per electrode were then isolated. Neuronal data along with behavioral events (occurrences of signals and performance of the animal) were stored on a PC for off-line analysis with a time resolution of $10 \mathrm{kHz}$.

In the following study, only trials where the response signal (RS) occurs at $1.7 \mathrm{~s}$ are considered. The expected signal (ES) corresponds to an eventually expected but not confirmed signal, i.e. at 1.2 s. Pairs 13 and 40 of the data set are considered here, as they were already treated in (Tuleau-Malot et al., 2014) and in (Riehle et al., 2000) with the Multiple Shift method (MS) of (Grün et al., 1999). This last analysis is also displayed 
on Figures 10 and 11 together with the methods described in the present article.

The $(\mathrm{TSC}+\mathrm{BH})$ method does not detect anything and is therefore not presented. The Permutation UE (P) method detects less windows than the (MTGAUE), (TSC) and (MS) methods. The above simulation study let us think that the extra detections of both (MTGAUE) and (TSC) may be False Positives, since both methods do not control the FDR as well as the Permutation UE method. However, the windows that are detected by the Permutation UE (P) method are still in adequation with the experimental or behavioral events. In particular, they still appear around the expected signal (ES) (blue vertical bar), which is completely coherent with the analysis made in (Riehle et al., 2000). Moreover (see Figure 11) the Permutation UE (P) method is able to detect also significant lack of coincidences as the original (MS) method. In Figure 11, there are also some windows that are detected by (P) but not by (TSC): this is also coherent with the simulations of Figure 7 showing that (TSC) is too conservative and may have as well too many false negatives.

\section{Discussion}

A UE method can be summarized in three steps:

(i) choose a coincidence count,

(ii) choose an approximation of the distribution of this count (or a function of this count) under independence to find correct $p$-values (in the sense that the corresponding tests control their False Positive (FP) rates),

(iii) combine the $p$-values for multiple testing on sliding windows. 
Our contribution to the steps (i) and (iii) is rather minor. As for the step (i), we indeed choose to use the delayed coincidence count introduced in (Tuleau-Malot et al., 2014), since it does not suffer from loss in synchrony detection. We here provide a fast and efficient algorithm to compute it with an even better run time than a basic algorithm for the binned coincidence count, using the sparsity of the signal. As for the step (iii), we straightforwardly adapt what has been proposed in (Tuleau-Malot et al., 2014). Note that up to an eventual logarithmic correction, this procedure mathematically guarantees a control of the False Discovery Rate as soon as the $p$-values are correct.

Our main contribution consists in a careful analysis of what has to be done to obtain correct $p$-values, that is of the step (ii).

A distribution free procedure. In this work, the only assumption that is made to obtain correct $p$-values is that the trials are independent and identically distributed. In particular no assumption is made on the underlying distribution of the spike trains. They can of course be homogeneous or inhomogeneous Poisson processes as shown in Figure 9 and Table 1. They can also be renewal processes in current or operational time (Nawrot, 2010; Pipa et al., 2013), have a conditional intensity as, for instance, Hawkes processes - see, for instance, Figure 9 and Table 1 or the simulations performed in (Albert et al., 2015) - or Wold processes (Pouzat \& Chaffiol, 2009), or they can have even more complicated structure of dependence with respect to their history - see, for instance, (Farkhooi et al., 2009, 2011). In fact they can be whatever one wants as long as one assumes that the distribution of the point processes is the same across the trials and that there is independence between the trials. All the mathematical material proving that 
they indeed can be "whatever" that is biologically reasonable, is contained in (Albert et al., 2015), where it has been shown that all the neuroscience models that we know are indeed satisfying the technical assumptions hidden behind those mathematical results. In short and for a non mathematical reader, it amounts to assume that each individual point process modeling a spike train cannot explode and cannot produce a gigantic number of spikes per unit of time, assumption which is always satisfied in practice thanks to biological constraints.

The centering issue. Under this i.i.d. assumption, we have focused on two distinct quantities: either $\mathbf{C}$, the total number of coincidence, whose expectation $c_{0}$ under independence $\left(H_{0}\right)$ is not known, or $\mathbf{U}$, a recentered count, which is obtained by subtracting to $\mathbf{C}$ an estimate $\hat{\mathbf{C}}_{0}$ of the unknown expectation under $\left(H_{0}\right)$ and which is therefore of zero mean under $\left(H_{0}\right)$. We have shown that, because we subtracted a random quantity, namely $\hat{\mathbf{C}}_{0}$, it is possible to obtain accurate approximation of the distribution of $\mathbf{U}$, the centered quantity, but the approximation does not hold for $\mathrm{C}$, the non centered one: this is the centering issue described in Section 3. In particular, the bootstrap principle, which is at the root of several surrogate data methods based on a shuffling of the trials, cannot be applied to non centered quantities It is therefore possible to see that the trialshuffling method introduced by (Pipa \& Grün, 2003; Pipa et al., 2003) performs poorly when directly applied to $\mathrm{C}$ (TSC) but that it very accurately approximates the desired distribution once $\mathbf{C}$ is correctly centered (TSU) (see, for instance, Figure 7). The same behavior is pointed out for the full bootstrap method, which is more classical from a statistical point of view. The permutation method is slightly better in the sense that, 
on the one hand, it does not suffer from the centering issue since the test based on the centered quantity $\mathbf{U}$ and the test based on the non centered quantity $\mathbf{C}$ are equivalent, and on the other hand, it is possible to mathematically guarantee the level whatever the number of trials (see the left part of Figure 7 where the corresponding $p$-values are under the diagonal even for $n=20$ trials). This is why we chose the permutation to complete the step (ii) of the UE method introduced in this article.

Practical implementation. Note that we used a Monte-Carlo approximation of the distribution in the provided complete algorithm, which has first been programmed and parallelized in $\mathrm{C}++$, and then interfaced with $\mathrm{R}$. In (Pipa et al., 2003) is given an exact algorithm when the trial-shuffling is applied to the coincidence count $\mathbf{C}$ directly. We did not follow this line of programming since this exact algorithm is quite long with respect to the Monte-Carlo algorithm when the number of simulations is 10000 (as used in the present work) and one can see on the bottom left of Figure 5 that the difference between both results (Monte-Carlo and exact algorithms) is not detectable at first glance. Simulations (in Figure 9 and Table 1) as well as a small real data set study show finally that the Permutation UE method offers more guarantee in terms of FDR than the methods of (Tuleau-Malot et al., 2014) and (Pipa \& Grün, 2003; Pipa et al., 2003) applied to the delayed coincidence count with a relatively comparable number of discoveries.

The i.i.d. assumption. The main point that remains to be discussed is the i.i.d. assumption in view of the classical sticking point in neuroscience: cross-trials nonstationarity. As shown on experimental studies (Arieli et al., 1996; Churchland et al., 2010; Avila-Akerberg \& Chacron, 2011), there is evidence of fluctuating ongoing ac- 
tivity in real neuronal networks, which leads to great cross-trials firing rate variability. Hence, and even if this variability seems sometimes to decrease with the stimulus, one needs to take it into account. Thus, the main question from a statistical point of view is: what does it mean for the distribution of the sample $\mathbb{X}_{n}$, that is the distribution of the observed data set ?

Several properties have been given in the literature as hints of cross-trials nonstationarity. In (Avila-Akerberg \& Chacron, 2011), a spike count having a positive variance is a result of "trial to trial variability". Yet with such a definition, i.i.d. homogenous Poisson processes, which actually have a spike count with positive variance, would be considered as cross-trials non-stationary, as well as any possible random model for spike trains. Other properties are expressed in terms of the Fano Factor $(F F)$, defined as the quotient of the variance of the spike count by the expectation of the spike count. In (Grün et al, 2003) and (Churchland et al., 2010) for instance, a $F F$ strictly larger than 1 is presented as a hint of cross-trials non-stationarity. But renewal processes with Gamma interspike interval (ISI) distributions may satisfy $F F>1$, which in fact only indicates that the processes are simply not homogeneous Poisson processes. In (Nawrot et al., 2008), a "measure" of non-stationarity across trials is given by the difference between the $F F$ and the variation of the ISI $\left(C V^{2}\right)$, which is the quotient of the variance of the ISI by the expectation of the ISI. Yet, in (Farkhooi et al., 2009; Nawrot, 2010), other models are constructed, with correlated ISI's, that satisfy $F F \neq C V^{2}$, and that are stationary across trial, this inequality only indicating that the processes are not renewal processes.

In view of all these studies, none of these properties, expected to be at least a 
hint of cross-trials non-stationarity, can be given as an exact definition of cross-trials non-stationarity. In our opinion, the best way to understand what is cross-trials nonstationarity is to carefully analyze the models that have been simulated to represent such a cross-trials non-stationarity in the above articles. From the simple one of (Grün et al, 2003) and simulated in Figure 8.C, to the very intricate one of (Farkhooi et al., 2011) through the statistical models used in (Ventura et al., 2005), one can see that they all share the principle of doubly stochastic processes. The article of (Churchland et al., 2011) is the one that maybe best formalizes this observation, as the cross-trials variability is explained from a "mixture of firing rate states", the firing rates changing "gradually during decision formation". This is what we tried to catch in a very simple way with the simulations of Figure 8.A and Figure 8.B. Following the description of (Churchland et al., 2011), there is a hidden variable $Y$, called an "intensity command", whose realization influences the parameters of the model for $X$ : typically, the firing rate of $X$ is a function of $Y$ whose value is fixed once $Y$ is given. The variable $Y$ may, for instance, model either the variation of depth in anesthesia, the changes in the level of attention of the animal or the degree of decision making. It can also be viewed as the stimulus in experiments that are subject to stimulus variability (Ben-Shaul et al., 2001) or as an oscillatory potential produced by a large non observed network of cells influencing both neurons (Kass et al., 2011).

From a probabilistic point of view, our interpretation is that cross-trials non-stationarity means that the distribution of the couple $X=\left(X^{1}, X^{2}\right)$ is not given intrinsically but is given conditionally to a certain random variable $Y$, that we call command variable hereafter in line with (Churchland et al., 2011). The question is then: can $Y$ be decom- 
posed in two independent "command" variables $Y^{1}$ and $Y^{2}$ that respectively govern the distributions of $X^{1}$ and $X^{2}$ or do we have a common command variable $Y$, that can be viewed as the "common source" of (Ben-Shaul et al., 2001)? In the first case, if $\left(X^{1}, X^{2}\right)$ are independent conditionally to $Y=\left(Y^{1}, Y^{2}\right)$, if the distribution of $X^{1}$ (respectively $X^{2}$ ) is only governed by $Y^{1}$ (respectively $Y^{2}$ ) and if $Y^{1}$ is independent on $Y^{2}$, then for all sets $A, B$,

$$
\mathbb{P}\left(X^{1} \in A, X^{2} \in B\right)=\mathbb{E}\left[\mathbb{P}\left(X^{1} \in A, X^{2} \in B \mid Y\right)\right]
$$

$$
=\mathbb{E}\left[\mathbb{P}\left(X^{1} \in A \mid Y\right) \mathbb{P}\left(X^{2} \in B \mid Y\right)\right] \text { by independence of }\left(X^{1}, X^{2}\right) \text { given } Y
$$

$$
=\mathbb{E}\left[\mathbb{P}\left(X^{1} \in A \mid Y^{1}\right) \mathbb{P}\left(X^{2} \in B \mid Y^{2}\right)\right]
$$

since there is no common command variable

$$
\begin{aligned}
& =\mathbb{E}\left[\mathbb{P}\left(X^{1} \in A \mid Y^{1}\right)\right] \mathbb{E}\left[\mathbb{P}\left(X^{2} \in B \mid Y^{2}\right)\right] \text { since } Y^{1} \text { is independent of } Y^{2} \\
& =\mathbb{P}\left(X^{1} \in A\right) \mathbb{P}\left(X^{2} \in B\right) .
\end{aligned}
$$

Hence in this case and despite the command variable $Y$, one is still under global independence between $X^{1}$ and $X^{2}$, that is $\left(H_{0}\right)$. As long as the command variable is i.i.d. across the trials, the distribution of $\mathbb{X}_{n}$ is therefore still the one of a $n$ i.i.d. sample satisfying $\left(H_{0}\right)$. This is exactly what happens in Figure 8.C, where the simulation scheme of (Grün et al, 2003; Grün, 2009) exactly satisfies this.

Since bootstrap methods are distribution free, they can in particular handle the fact that the distribution of $X$ is described via this doubly stochastic process. The only thing that matters is whether there is still global (unconditional) independence between $X^{1}$ and $X^{2}$. We believe that the explanation for the bad behavior of the trial-shuffling described in (Grün et al, 2003; Grün, 2009) is not cross-trials non-stationarity but a 
centering defect, which can be seen via the behavior of (TSC) versus (TSU) on Figure 8.C. It is indeed possible that when using the binned coincidence count instead of the delayed coincidence count, (TSC) goes from too conservative as on Figure 8.C to not enough conservative as shown in the study of (Grün et al, 2003; Grün, 2009). In both cases, (TSC) does not reproduce the right distribution under $\left(H_{0}\right)$ because the quantity at hand is not correctly centered, but once this is corrected, (TSU) is perfectly able to give correct $p$-values even in this cross-trials non-stationary case.

The same explanation holds for Figure 8.B. In this case, the command variable is the index of the trial but it influences only $X^{1}$ and not $X^{2}$, so we are exactly in the same set-up as without any common command variable: the $p$-values behave exactly as usual under $\left(H_{0}\right)$. However in Figure 8.A, a common command variable (again the index of the trial) governs both distributions: the $p$-values behave exactly as under $\left(H_{1}\right)$ in Figure 7. Note that it is actually reasonable to reject independence here: indeed (7) does not hold and the variables $X^{1}$ and $X^{2}$ are globally dependent here, since there is definitely a common command variable. A similar set-up of common command variable can be viewed in the models of conditional dependence proposed by (Ventura et al., 2005) and (Kass et al., 2011).

To conclude, what the surrogate methods based on a shuffling of the trials can do with respect to cross-trials non-stationarity is also to detect whether there is a common command variable or not. In particular, if $X^{1}$ and $X^{2}$ are independent conditionally to the common command variable $Y$ and do not present any "fine temporal coordination of spikes in neuronal preprocessing", as stated in (Grün et al, 2003), the test is still likely to reject the independence assumption. Yet, this is not a False Positive with respect to 
the statistical meaning of the test. Indeed, in this situation the spike trains $X^{1}$ and $X^{2}$ are correlated since they are not globally independent. However, they are conditionally independent once the command variable is fixed and in this sense they do not really present any synchrony. This kind of distinction between correlation and synchrony was already underlined and discussed on cross-correlograms by Brody (1999a,b).

Finally, one could wonder what is really assumed by i.i.d. trials. The independence between trials is, in our opinion, not really an issue since the trials are usually sufficiently far apart in time. The main assumption is therefore the identical distribution. As explained above, cross-trials non-stationarity interpreted as a command variable phenomenon does not contradict this assumption. We can even not imagine how this assumption can be defective in practice. Even in the extreme case where half of the trials would be sampled from an anesthetized animal and the other half from a non anesthetized animal, considering the presence or not of anesthesia as a command variable lead to i.i.d. trials from a mixture point of view.

This naturally leads to the following completely open question. Is the global independence property really the assumption that the neuroscientists want to test?

On the one hand, in (Churchland et al., 2011), it is stated that "variance itself can be diagnostic of neural computation". We interpret this in the present framework as follows: if one is able to detect a common command variable (not known before hand), then one is able to detect "neural computation". This line is totally in accordance with the discussion of (Ben-Shaul et al., 2001) where global dependence can be viewed as the presence of an "internal variable" when "the variability of all relevant stimuli or actions has been accounted for" by the experimental design and therefore when the test 
has been applied only to trials that are homogeneous with respect to this experimental design. Hence global independence may have a meaning in neuroscience.

On the other hand, a more precise description of the dependence may be needed. For instance, Hawkes processes allow to model local independence. For instance, if the command variable is the spike train of a third observed neuron, it is eventually possible via the methods of (Hansen et al., 2015; Reynaud-Bouret et al., 2013) and under a Hawkes distribution assumption, to see whether this third neuron influences both $X^{1}$ and $X^{2}$ with or without direct (local) dependence between $X^{1}$ and $X^{2}$. In the same line, the works of (Ventura et al., 2005; Kass et al., 2011) give another precise model of conditional independence that can be tested. Up to our knowledge, however, there is no distribution free method that would be able to assess this, in particular if the command variable is hidden.

Therefore, contrary to what is currently believed, the present statistical study shows that surrogate data methods based on a shuffling of the trials can behave properly under cross-trials non-stationarity if they are applied to correctly centered quantities and if one wishes to detect global dependence. The other popular surrogate method based on dithering (Louis et al., 2010b) is much more difficult to study from a mathematical point of view, principally because, unlike bootstrap methods, there is no general mathematical theory explaining why moving individual spikes would mimic the overall distribution of the coincidence count or any centered version of it under independence. One possible guess, which is maybe far fetched, is that dithering, as a much more local surrogate data procedure, may be able somehow to detect local and not global dependence, if one can correctly tune it. 
Another open question, which seems much more achievable, is to adapt those bootstrap procedures to more than two neurons. Indeed, delayed coincidence counts have already been introduced in this case in (Chevallier \& Laloë, 2015) and similar bootstrap procedures have been developed for more than two real valued variables in the precursor work of (Romano, 1989).

\section{Acknowledgments}

We first of all want to thank A. Riehle, leader of the laboratory in which the data used in this article were previously collected. We are very grateful to F. Grammont, who notably recorded the data, for fruitful and stimulating discussions. We also warmly thank Gilles Blanchard for all his relevant suggestions, and both referees for their comments, which really helped us to improve the present manuscript. This work was granted access to the HPC and visualization resources of "Centre de Calcul Interactif" hosted by the University of Nice Sophia-Antipolis. It was partly supported by the french Agence Nationale de la Recherche (ANR 2011 BS01 01001 projet Calibration), by the PEPS BMI 2012-2013 Estimation of dependence graphs for thalamo-cortical neurons and multivariate Hawkes processes and by the interdisciplinary axis MTC-NSC of the University of Nice Sophia-Antipolis. The PhD grant of M. Albert is funded by the PACA french region. 


\section{References}

Arieli, A., Sterkin, A., Grinvald, A., \& Aertsen, A. (1996) Dynamics of Ongoing activity: explanation of the large variability in evoked cortical responses. Science, $273,1868-1871$.

Aertsen, A.M., Gerstein, G.L., Habib, M.K., \& Palm, G. (1989) Dynamics of neuronal firing correlation: modulation of "effective connectivity". Journal of Neurophysiology, 61(5), 900-917.

Albert, M., Bouret, Y., Fromont, M., \& Reynaud-Bouret, P. (2015) Bootstrap and permutation tests of independence for point processes. to appear in Annals of Statistics.

Avila-Akerberg, O., \& Chacron, M. J. Nonrenewal spike trains statistics: causes and functional consequences on neural coding. Exp. Brain Res., 210, 353-371.

Benjamini, Y., \& Hochberg, Y. (1995) Controlling the false discovery rate: a practical and powerful approach to multiple testing. Journal of the Royal Statistical Society, Series B, 57(1), 289-300.

Benjamini, Y., \& Yekutieli, D. (2001) The control of the false discovery rate in multiple testing under dependency. Annals of Statistics, 29(4), 1165-1188.

Ben-Shaul, Y., Bergman, H., Ritov, H., \& Abeles, M. (2001) Trial to trial variability in either stimulus or action causes apparent correlation and synchrony in neuronal activity. Journal of Neuroscience Methods, 111, 99-110.

Bickel, P. J., \& Freedman, D.A. (1981) Some asymptotic theory for the bootstrap. Ann. Statist., 9(6), 1196-1217. 
Brody, C. D. (1999a) Correlations without synchrony. Neural Computation, 11, 15371551.

Brody, C. D. (1999b) Disambiguating different covariation types. Neural Computation, 11, 1527-1535.

Chevallier, J., \& Laloë, T. (2015) Detection of dependence patterns with delay. to appear in Biometrical Journal.

Churchland, A. K., Kiani, R., Chaudhuri, R., Wang, X.-J., Pouget, A., \& Shadlen, M. N. (2011) Variance as a signature of neural computations during decision making. Neuron, 69, 818-831.

Churchland, M. M., Yu, B. M., Cunningham, J. P., Sugrue, L. P., Cohen, M. R., Corrado, G. S., Newsome, W. T., Clark, A. M., Hosseini, P., Scott, B. B., Bradley, D. C., Smith, M. A., Kohn, A., Movshon, J. A., Armstrong, K. M., Moore, T., Chang, S. W., Snyder, L. H., Lisberger, S. G., Priebe, N. J., Finn, I. M., Ferster, D., Ryu, S. I., Santhanam, G., Sahani, M., \& Shenoy, K. V. (2010) Stimulus onset quenches neural variability: a widespread cortical phenomenon. Nature Neursocience, 13(3), 369-378.

Daley, D.J., \& Vere-Jones, D. (2005) An introduction to the theory of point processes. Springer series in statistics Volume I.

Denker, M., Wiebelt, B., Fliegner, D., Diesmann, M., \& Morrison, A. (2010) Practically Trivial Parallel Data Processing in a Neuroscience Laboratory. In Analysis of Parallel Spike Trains, Grün, S., \& Rotter, S., Springer Series in Computational Neuroscience. 
Farkhooi, F., Froese, A., Muller, E., Menzel, R., \& Nawrot, M. P. (2013) Cellular adaptation facilitates sparse and reliable coding in sensory pathways. PLOS Comp. Biol., 9(10), e1003251.

Farkhooi, F., Muller, E.,\& Nawrot, M. P. (2011) Adaptation reduces variability of neuronal population code. Physical Review E, 83, 050905.

Farkhooi, F., Strube-Bloss, M. F., \& Nawrot, M. P. (2009) Serial correlation in neural spike trains: Experimental evidence, stochastic modeling, and single neuron variability. Physical Review E, 79, 021905.

Giné, E. (1997) Lectures on some aspects of the bootstrap. Lecture Notes in Math 1665, 37-152. Ecole d'été de Probabilités de Saint-Flour, XXVI-1996.

Grammont, F., \& Riehle, A. (2003) Spike synchronisation and firing rate in a population of motor cortical neurons in relation to movement direction and reaction time. Biological Cybernetics, 88, 360-373.

Grün, S. (1996) Unitary joint-events in multiple-neuron spiking activity: Detection, significance and interpretation. Thun: Verlag Harri Deutsch.

Grün, S. (2009) Data-driven Significance estimation for precise spike correlation. $J$. Neurophysiol., 101, 1126-1140.

Grün, S., Diesmann, M., \& Aertsen, A.M. (2002) Unitary events in multiple singleneuron activity: I. Detection and significance Neural Computation, 14(1), 43-80.

Grün, S., Diesmann, M., \& Aertsen, A.M. (2002) Unitary events in multiple singleneuron activity: II. Nonstationary data Neural Computation, 14(1), 81-119. 
Grün, S., Diesmann, M., \& Aertsen, A.M. (2010) Unitary Events Analysis. In Analysis of Parallel Spike Trains, Grün, S., \& Rotter, S., Springer Series in Computational Neuroscience.

Grün, S., Diesmann, M., Grammont, F., Riehle, A., \& Aertsen, A. (1999) Detecting unitary events without discretization in time. Journal of Neuroscience methods 94.

Grün, S., Riehle, A., \& Diesmann, M. (2003) Effect of cross-trial nonstationarity on joint-spike events. Biol. Cybern., 88, 335-351.

Gütig, R., Aertsen, A.M., \& Rotter, S. (2001). Statistical Significance of Coincident Spikes: Count-Based Versus Rate-Based Statistics. Neural Computation, 14, 121153.

Hansen, N.R., Reynaud-Bouret, P., \& Rivoirard, V. (2015). Lasso and probabilistic inequalities for multivariate point processes. Bernoulli, 21(1), 83-143.

Hoeffding, V. (1952) The large sample power of tests based on permutation of the observations. The Annals of Mathematical Statistics, 3(2), 169-192.

Kass, R.E., Kelly, R. C., \& Loh, W.-L. (2011) Assessment of synchrony in multiple neural spike trains using loglinear point process models. Annals of Applied Statistics, 5(2B), 1262-1292.

Kilavik, B.E., Roux, S., Ponce-Alvarez, A. Confais, J. Grün, S., \& Riehle, A.(2009) Long-term modifications in motor cortical dynamics induced by intensive practice. Journal of Neuroscience, 29(40), 12653-12663. 
Litwin-Kumar, A., \& Doiron, B. (2012) Slow dynamics and high variability in balanced cortical networks with clustered connections. Nature Neuroscience, 15(11), 14981505.

Louis, S., Borgelt, C., \& Grün, S. (2010) Generation and Selection of Surrogate Methods for Correlation Analysis. In Analysis of Parallel Spike Trains, Grün, S., \& Rotter, S., Springer Series in Computational Neuroscience.

Louis, S., Gerstein, G. L., \& Diesmann, M. (2010) Surrogate spike train generation through dithering in operational time. Frontiers in Computational Neuroscience, 4 (article 127).

Nawrot, M. P. (2010) Analysis and interpretation of interval and count variability in neural spike trains. In Analysis of Parallel Spike Trains, Grün, S., \& Rotter, S., Springer Series in Computational Neuroscience.

Nawrot, M. P., Boucsein, C., Molina, V. R., Riehle, A., Aertsen, A., \& Rotter, S. (2008) Measurement of variability dynamics in cortical spike trains. Journal of Neuroscience Methods, 169, 374-390.

Perkel, D.H., Gernstein, G.L., \& Moore, G.P. (1967) Neuronal spike trains and stochastic point processes. Biophysical Journal, 7, 419-440.

Pipa, G., Diesmann, M., \& Grün, S. (2003) Significance of joint-spike events based on trial-shuffling by efficient combinatorial methods. Complexity, 8(4), 1-8.

Pipa, G., \& Grün, S. (2003) Non-parametric significance estimation of joint-spike events by shuffling and resampling. Neurocomputing, 52-54, 31-37. 
Pipa, G., Grün, S., \& van Vreeswijk, C. (2013). Impact of Spike Train Autostructure on Probability Distribution of Joint Spike Events. Neural Computation, 25, 1123-1163.

Pouzat, C., \& Chaffiol, A. (2009) Automatic spike train analysis and report generation. An implementation with R, R2HTML and STAR. Journal of Neuroscience Methods, $181,119-144$.

Reynaud-Bouret, P., Rivoirard, V., \& Tuleau-Malot, C. (2013) Inference of functional connectivity in Neurosciences via Hawkes processes. 1st IEEE Global Conference on Signal and Information Processing, Austin, Texas.

Riehle, A., Grammont, F., Diesmann, M., \& Grün, S. (2000) Dynamical changes and temporal precision of synchronised spiking activity in monkey motor cortex during movement preparation. Journal of Physiology, 94, 569-582.

Riehle, A., Grammont, F., \& MacKay, A. (2006) Cancellation of a planned movement in monkey motor cortex. Neuroreport, 17(3), 281-285.

Romano, J.P. (1989) Bootstrap and Randomization Tests of some Nonparametric Hypotheses. Ann. Statist. 17(1), 141-159.

Romano, J.P., \& Wolf, M. (2005) Exact and approximate step-down methods for multiple hypothesis testing. J. Amer. Statist. Assoc., 100, 94-108.

Singer, W. (1993) Synchronization of cortical activity and its putative role in information processing and learning. Annual Review of Physiology, 55, 349-374.

Tuleau-Malot, C., Rouis, A., Grammont, F., \& Reynaud-Bouret, P. Multiple Tests 
based on a Gaussian Approximation of the Unitary Events method with delayed coincidence count. Neural Computation, 26(7), 1408-1454.

Ventura, V. (2010) Bootstrap tests of hypotheses. In Analysis of Parallel Spike Trains, Grün, S., \& Rotter, S., Springer Series in Computational Neuroscience.

Ventura, V., Cai, C., \& Kass, R.E. (2010) Trial-to-trial variability and its effect on time-varying dependency between two neurons. Journal of Neurophysiology, 94, $2928-2939$. 


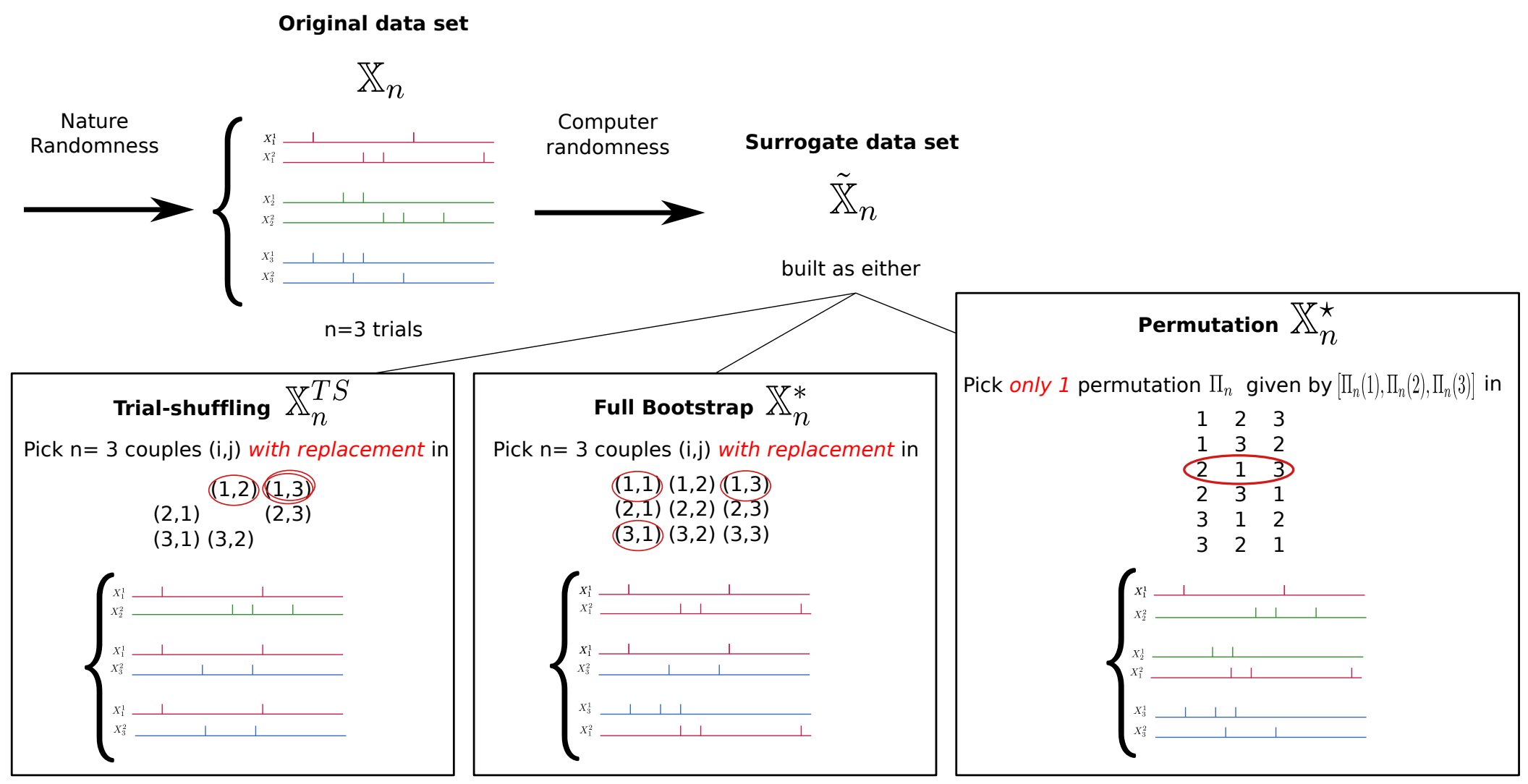

Unconditional distribution: all possible choices of both Nature and Computer randomness

Conditional distribution: 1 fixed original data set (Nature randomness), all possible choices of Computer randomness

Figure 4: Schematic view of the three bootstrap procedures. Note in particular that $n$ draws with replacement are necessary for the trial-shuffling and the full bootstrap approach, whereas only one draw of one permutation is necessary for the permutation approach. Note also that it is perfectly possible that a surrogate data set done by trialshuffling or full bootstrap approaches may perfectly pick twice the same trial and at the same time leave out one or more of the original trials, whereas the permutation is always exhaustive in this sense. Such typical draws are given by the red circles, leading to the given surrogate data set for each method. Finally note that the unconditional distribution let both randomness (Nature and Computer) vary and that this is not realistic since in practice we have only one original data set. This is why the conditional distribution is the one that can be simulated via a computer for a given observation. 

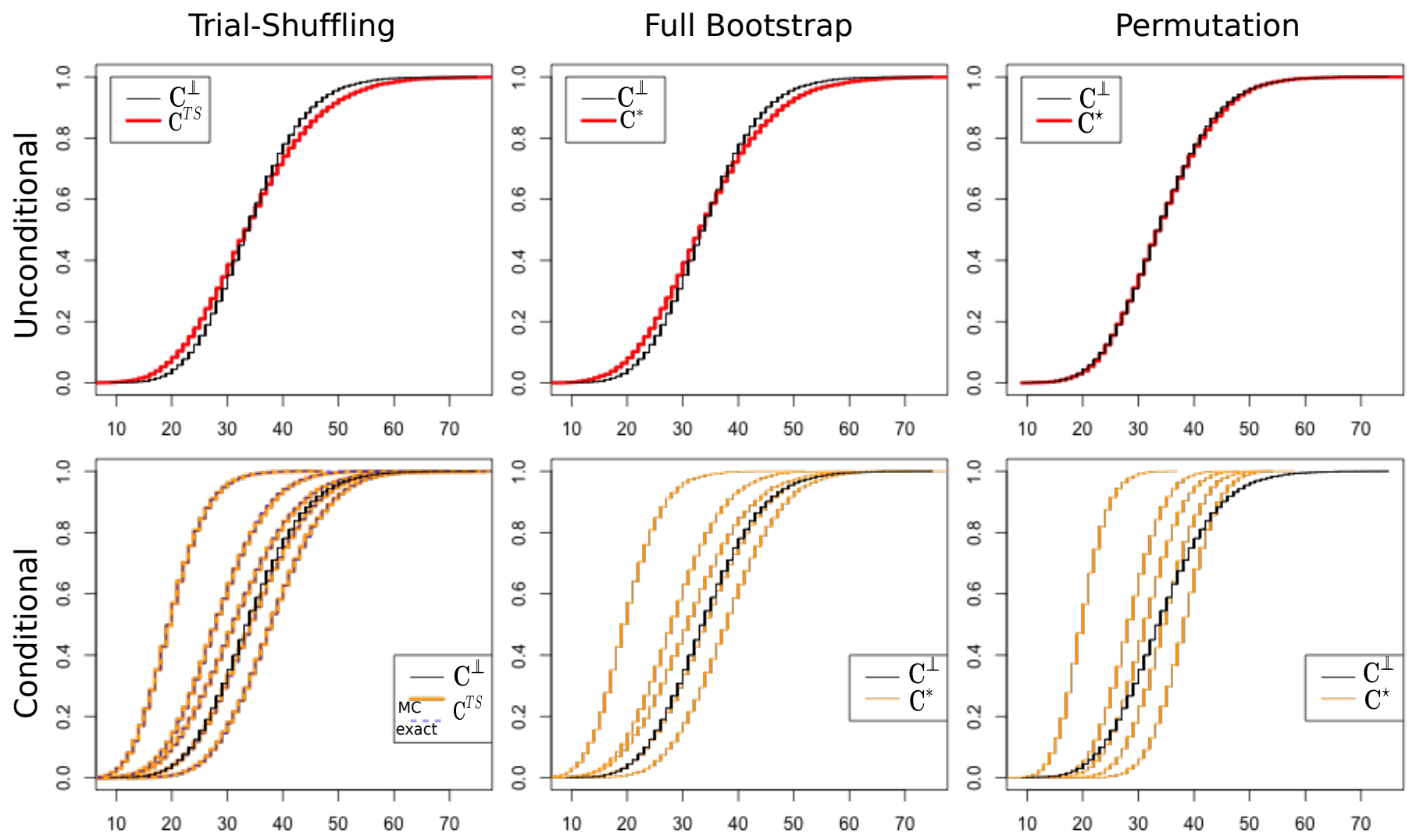

Figure 5: The unconditional distribution and conditional distributions of $\mathbf{C}$ under $\left(H_{0}\right)$. C.d.f. of $\mathbf{C}\left(\mathbb{X}_{n}^{\Perp}\right)$ and (for the first line) of $\mathbf{C}^{T S}=\mathbf{C}\left(\mathbb{X}_{n}^{T S}\right)$, of $\mathbf{C}^{*}=\mathbf{C}\left(\mathbb{X}_{n}^{*}\right)$ and of $\mathbf{C}^{\star}=\mathbf{C}\left(\mathbb{X}_{n}^{\Pi_{n}}\right)$ obtained from 10000 simulations of $n=20$ trials of two independent Poisson processes of firing rate $30 \mathrm{~Hz}$ on a window of length $0.1 \mathrm{~s}$ with $\delta=0.01 \mathrm{~s}$. On the second line, in addition to the c.d.f. of $\mathbf{C}\left(\mathbb{X}_{n}^{\Perp}\right)$, five observations of $\mathbb{X}_{n}=\mathbb{X}_{n}^{\Perp}$ have been simulated in the same set-up and given these observations, the conditional c.d.f. have been approximated by simulating 10000 times the extra-randomness corresponding to $\tilde{\mathbb{X}}_{n}$. For the trial-shuffling, in addition to this approximate Monte-Carlo method (MC), the exact conditional c.d.f. has been obtained thanks to the algorithm of (Pipa et al., 2003). 


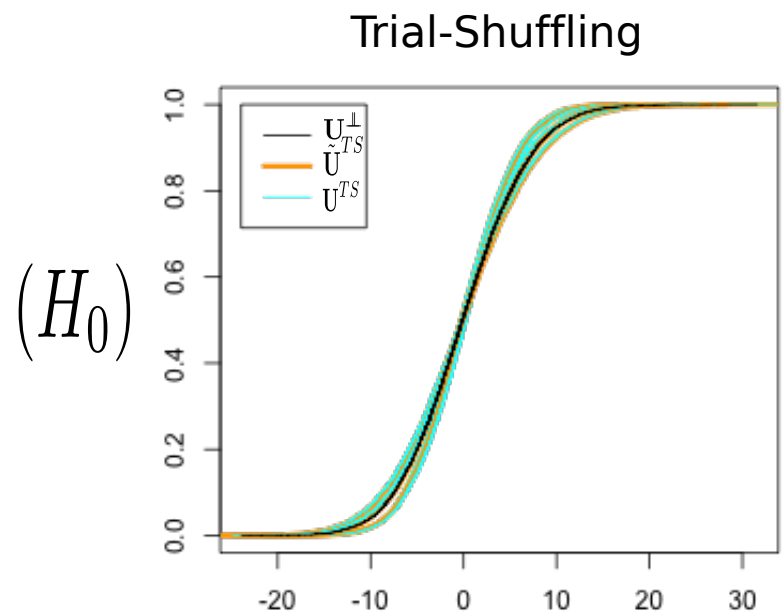

Full Bootstrap
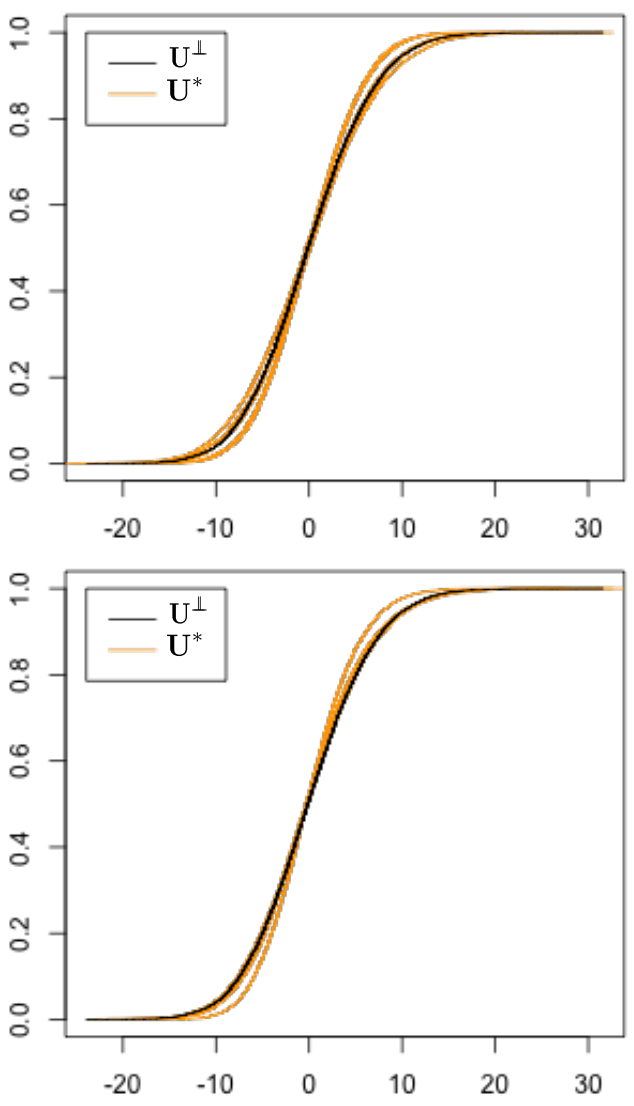

Permutation
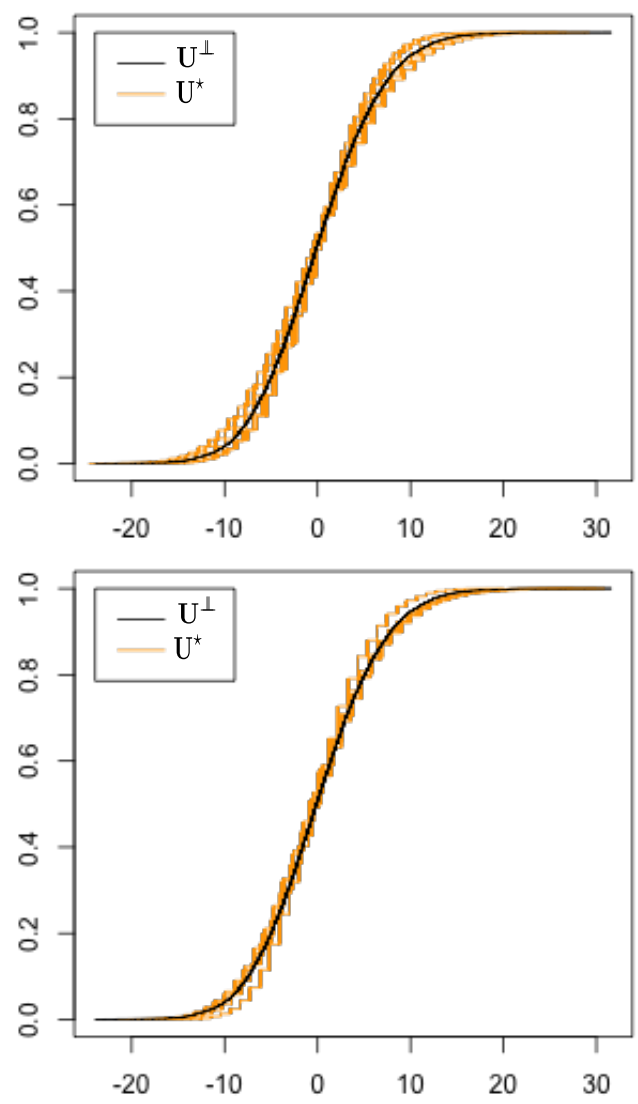

Figure 6: Conditional distribution of $\mathbf{U}\left(\tilde{\mathbb{X}}_{n}\right)$ (or its recentered version $\tilde{\mathbf{U}}^{T S}$ for the Trial-Shuffling) given $\mathbb{X}_{n}$. Cumulative distribution functions of $\mathbf{U}^{\Perp}=\mathbf{U}\left(\mathbb{X}_{n}^{\Perp}\right)$ in black, obtained by simulation as in Figure 5. For the first line, under $\left(H_{0}\right)$, five observations of $\mathbb{X}_{n}^{\Perp}$ in the same set-up have been fixed and given these observations, the conditional c.d.f. of $\mathbf{U}^{T S}=\mathbf{U}\left(\mathbb{X}_{n}^{T S}\right)$, of $\tilde{\mathbf{U}}^{T S}=\mathbf{U}^{T S}+\mathbf{U}^{\text {obs }} / n$, of $\mathbf{U}^{*}=\mathbf{U}\left(\mathbb{X}_{n}^{*}\right)$ and of $\mathbf{U}^{\star}=$ $\mathbf{U}\left(\mathbb{X}_{n}^{\Pi_{n}}\right)$ have been obtained as in Figure 5. For the second line, five observations of $\mathbb{X}_{n}$, simulated under $\left(H_{1}\right)$ with marginals equal to the ones of the first line but satisfying $X^{1}=X^{2}$, have been simulated and conditional c.d.f. are obtained in the same way as above. 

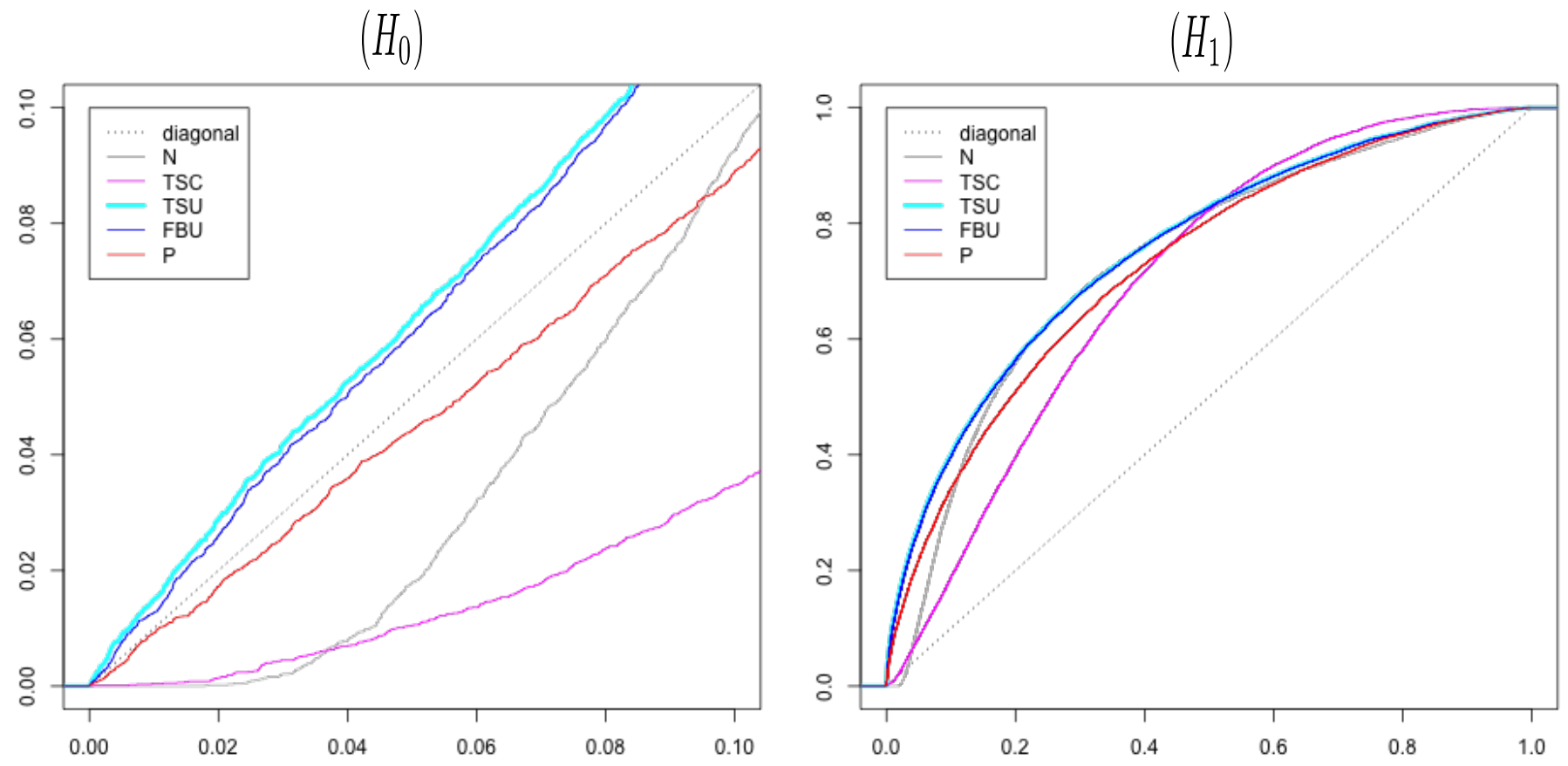

Figure 7: Distribution of the $p$-values for the different tests. C.d.f. under both $\left(H_{0}\right)$ and $\left(H_{1}\right)$ of the $p$-values for the five tests: naive $(\mathrm{N})$, Trial-Shuffling version $\mathbf{C}$ (TSC), Trial-Shuffling version U (TSU), Full Bootstrap version U (FBU), and Permutation (P). Under $\left(H_{0}\right)$, the c.d.f. are obtained by simulations done as in Figure 5; the c.d.f. are then plotted only for small $p$-values $(\leq 0.1)$. Under $\left(H_{1}\right)$, the couple $\left(X^{1}, X^{2}\right)$ is constructed by injection (Grün et al., 2010; Tuleau-Malot et al., 2014), i.e. as $\left(N^{1} \cup N^{i n j}, N^{2} \cup N^{i n j}\right)$ where $\left(N^{1}, N^{2}\right)$ are two independent Poisson processes of firing rate $27 \mathrm{~Hz}$ on a window of length $0.1 \mathrm{~s}$ and where $N^{i n j}$ is a common Poisson process of firing rate $3 \mathrm{~Hz}$; once again, 20 i.i.d. trials are simulated 10000 times to obtain the corresponding c.d.f. with $\delta=0.01 \mathrm{~s}$ 


\section{A: Drift on both X1 and X2}

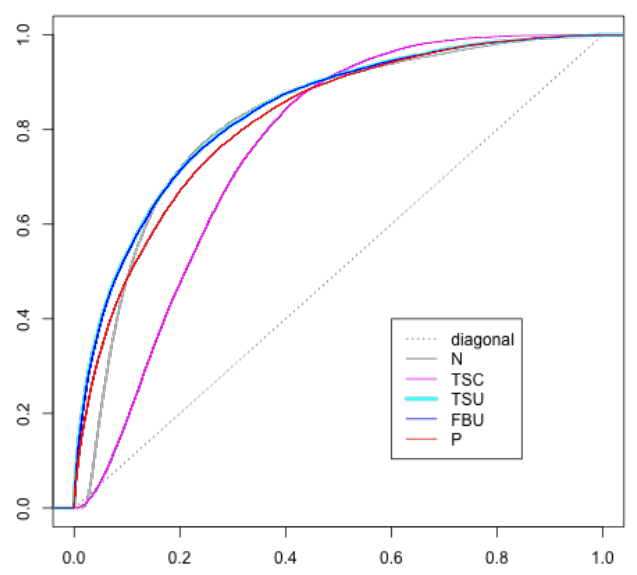

B: Drift on X1, No drift on X2

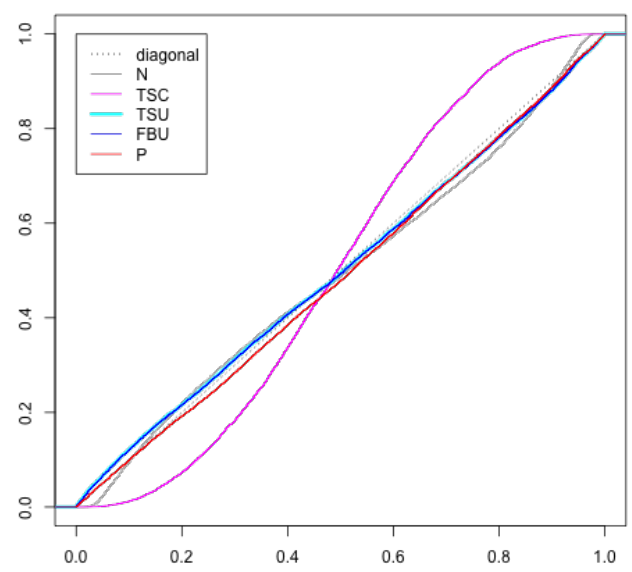

C: Hidden command

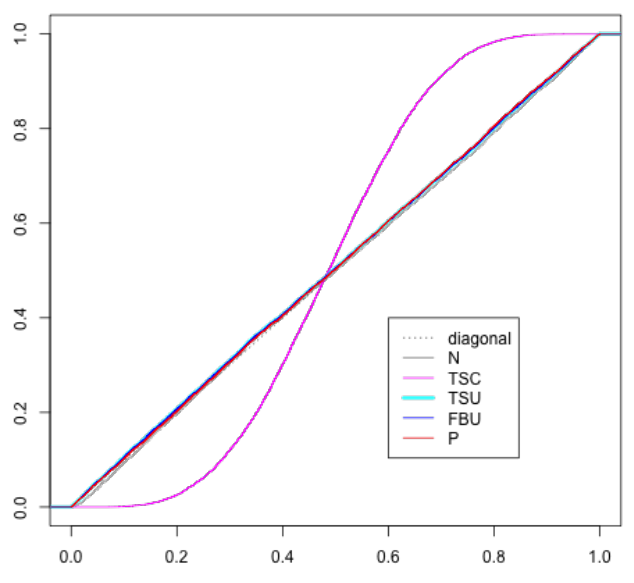

Figure 8: Distribution of the $p$-values for the different tests under varying firing rates.

C.d.f. of the $p$-values for the five tests: naive (N), Trial-Shuffling version $\mathbf{C}$ (TSC), Trial-Shuffling version U (TSU), Full Bootstrap version U (FBU), and Permutation (P) computed over 10000 simulations. In $\mathbf{A}, n=20$ trials are drawn, the firing rates are constant on each trial and they are regularly increasing, from trial 1 to trial 20 , from respectively $10 \mathrm{~Hz}$ to $100 \mathrm{~Hz}$ for $X^{1}$ and from $10 \mathrm{~Hz}$ to $50 \mathrm{~Hz}$ for $X^{2}$. Once the rates fixed, both spike trains in each trial are independent homogeneous Poisson processes of the prescribed rates. The length of the interval $[a, b]$ is $0.1 \mathrm{~s}$ and $\delta=0.01 \mathrm{~s}$. In $\mathbf{B}$, the same set-up is taken except that the firing rate of $X^{2}$ is fixed equal to $50 \mathrm{~Hz}$. In $\mathbf{C}$, the simulation set up of (Grün, 2009) is taken: 100 trials of 1s duration and in each trial $i$ and each component $j$ is independently drawn (i) first, a hidden command variable, $Y_{i}^{j}$, which is here a Bernoulli variable of parameter 0.7 (ii) the spike train $X_{i}^{j}$ is then simulated as a homogeneous Poisson process of firing rate $30 \mathrm{~Hz}$, if $Y_{i}^{j}=1$ and of firing rate $90 \mathrm{~Hz}$, if $Y_{i}^{j}=0$. 


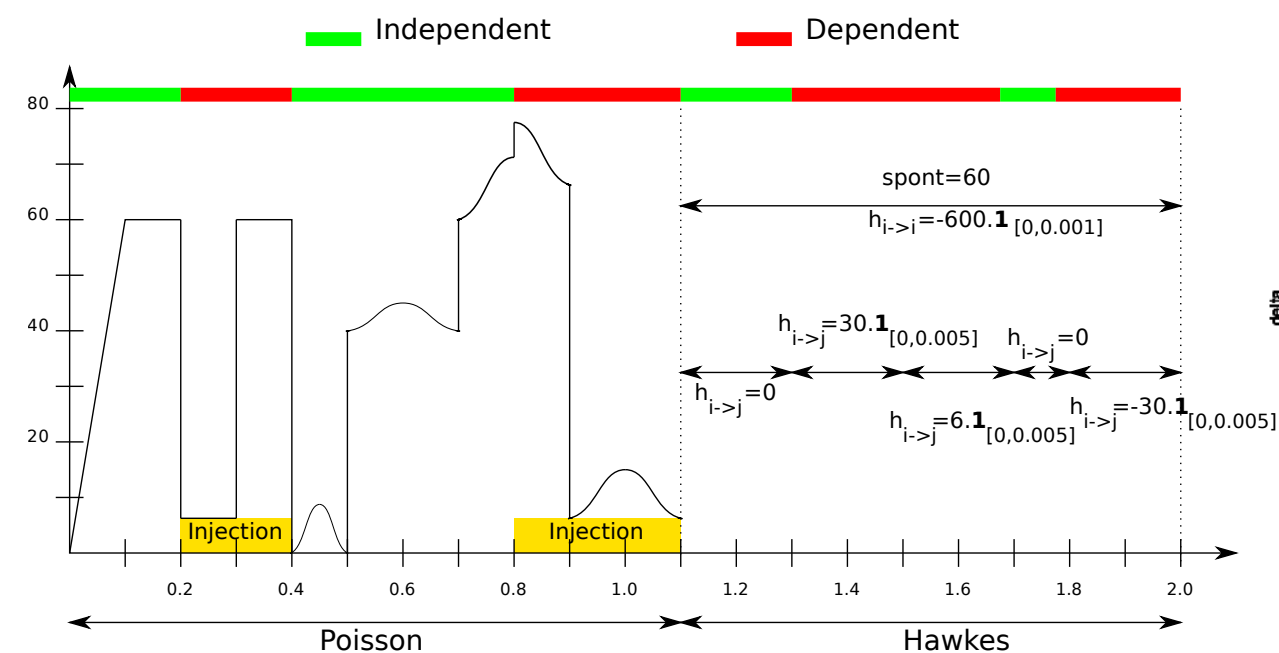

A: Description of Experiment 1

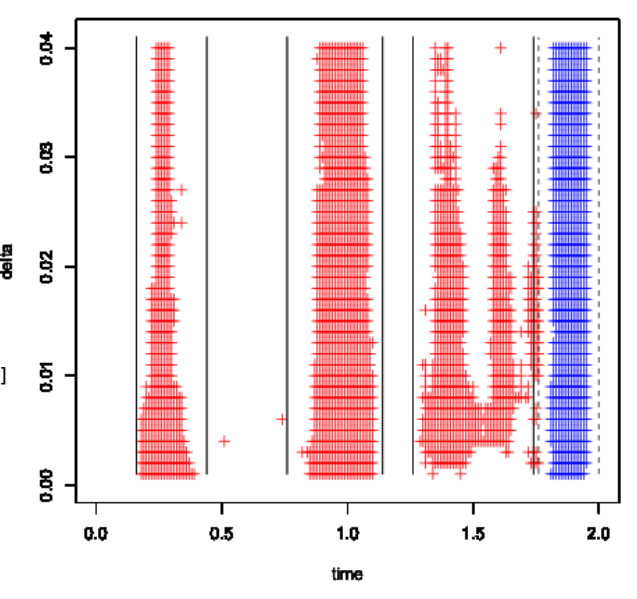

B: Result of the permutation method

Figure 9: Multiple tests. 9.A: description of Experiment 1. In the Poisson part, the intensity of both Poisson processes is plotted. The injection component corresponds to the part of a shared Poisson process which is injected in both processes corresponding to $X^{1}$ and $X^{2}$, as explained in Figure 7. In the Hawkes part (see (Tuleau-Malot et al., 2014) for a complete description), formulas for the spontaneous parameters and both self interaction $h_{i \rightarrow i}$ and cross interaction $h_{i \rightarrow j}$ functions are given. 9.B: results of the Permutation UE method $(B=10000, q=0.05$ ) performed on 191 overlapping windows of the form $[a, a+0.1]$ for $a$ in $\{0,0.01, \ldots, 1.9\}$ on one run of simulation for 50 trials of Experiment 1. A red (resp. blue) cross is represented at the center of the window when it is detected by a $p_{W}^{+}\left(\right.$resp. $\left.p_{W}^{-}\right)$. Each horizontal line corresponds to a different $\delta$ in $\{0.001,0.002, \ldots 0.04\}$. The black vertical lines delimit the regions where the independence hypothesis is not satisfied: plain for positive dependence (i.e. where $\mathbf{C}^{\text {obs }}$ should be too large), and dashed for negative dependence (i.e. where $\mathbf{C}^{\text {obs }}$ should be too small). 
MS

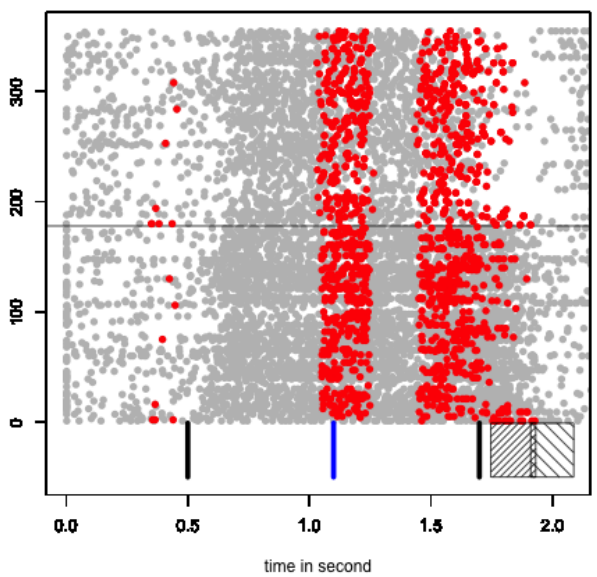

TSC

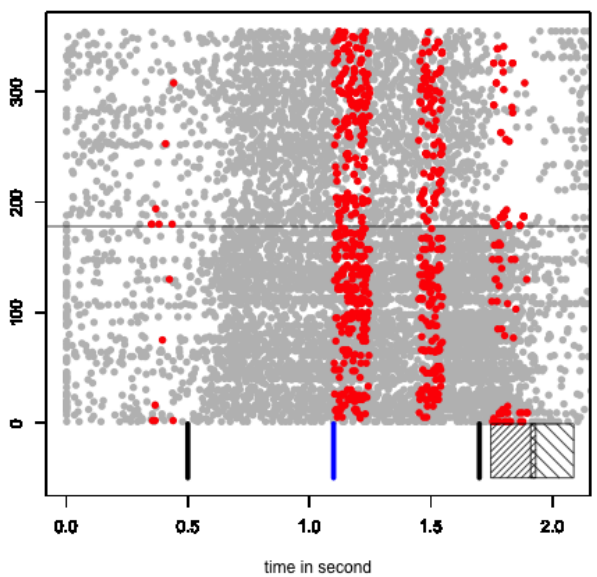

MTGAUE

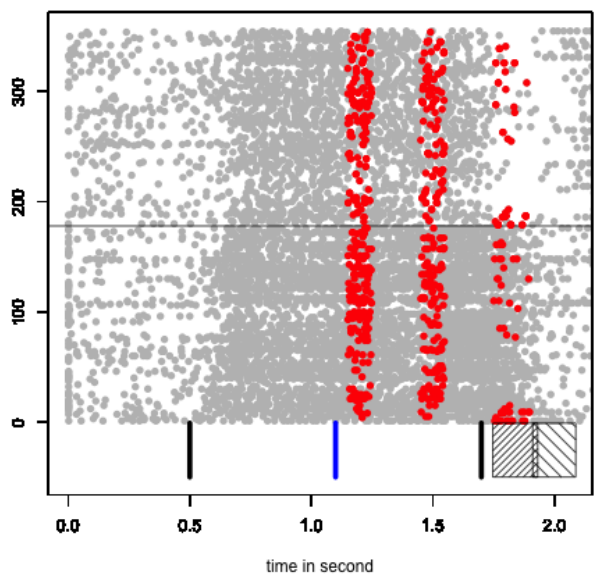

$\mathbf{P}$

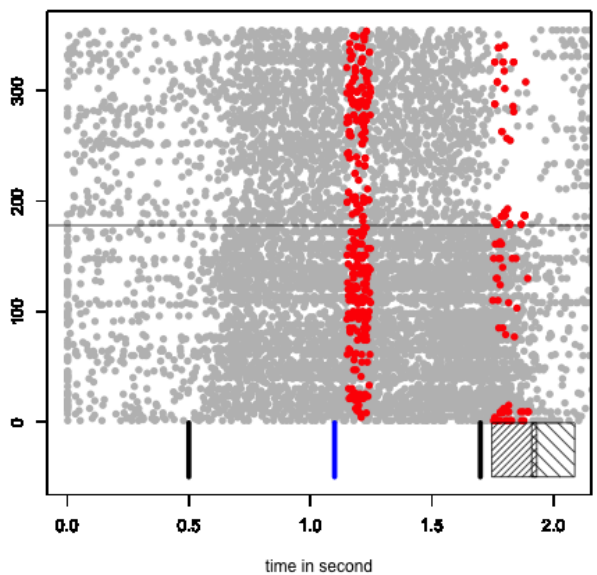

Figure 10: Raster plots of the pair of neurons 13. In red the Unitary Events where the coincidence count is significantly too large for the three methods (MTGAUE, TSC and P) presented in Table 1 and for the Multiple Shift method (MS), with $\delta=0.02 \mathrm{~s}$ and $B=10000$, on overlapping windows of the form $[a, a+0.1]$ for $a$ in $\{0,0.05, \ldots, 1.95\}$. No interval was detected for a significantly too small coincidence count. Signs on bottom corresponds to behavioral events. The first black vertical bar corresponds to the preparatory signal (PS), the blue vertical bar to the expected signal (ES), the second black vertical bar to the response signal (RS). The first hatched box corresponds to the interval [mean reaction time (RT) minus its standard deviation, mean reaction time (RT) plus its standard deviation], the second hatched box corresponds to the same thing but for the movement time (MT). 

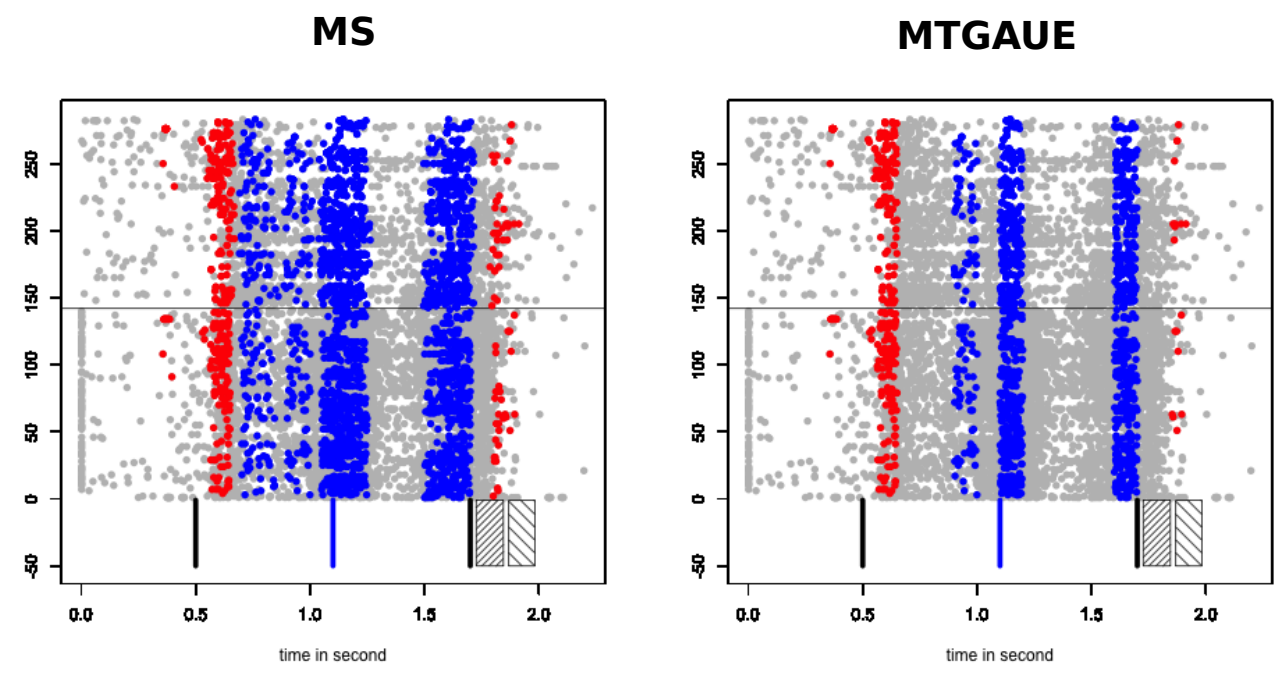

TSC
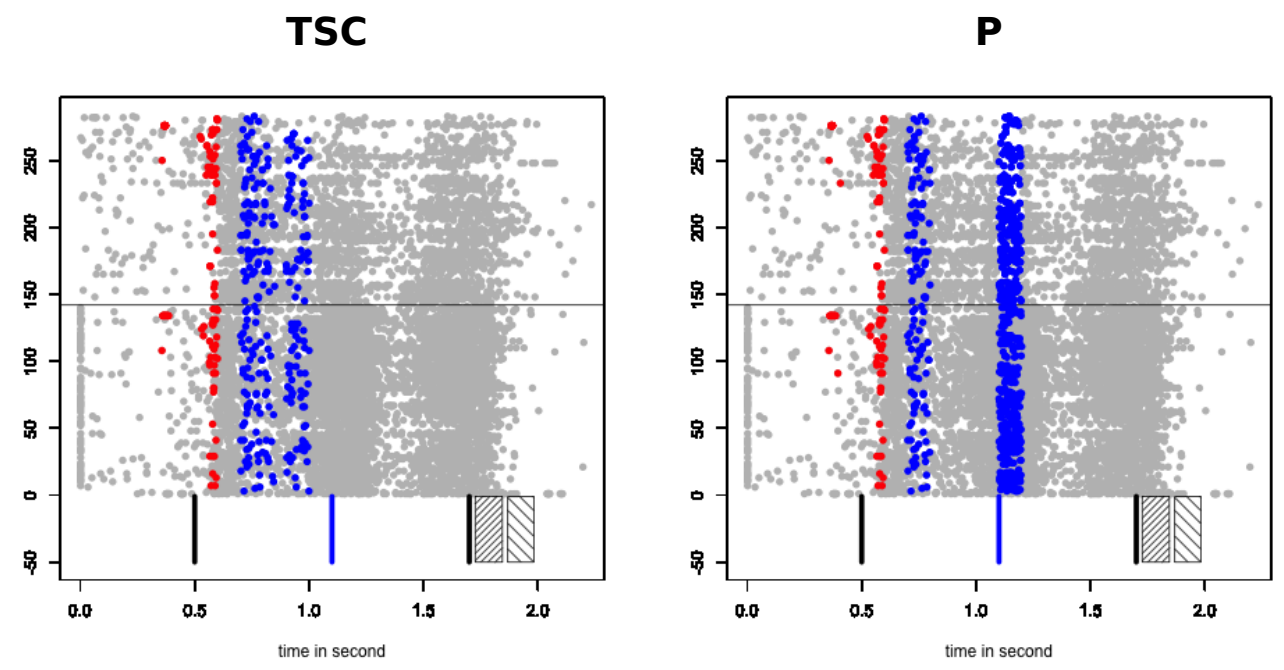

Figure 11: Raster plots of the pair of neurons 40. In red the Unitary Events where the coincidence count is significantly too large for the three methods (MTGAUE, TSC and P) presented in Table 1 and for the Multiple Shift method (MS), with $\delta=0.02 \mathrm{~s}$ and $B=10000$, on overlapping windows of the form $[a, a+0.1]$ for $a$ in $\{0,0.05, \ldots, 2.1\}$. In blue the Unitary Events where the coincidence count is significantly too small with the same convention. Signs on bottom corresponds to behavioral events as described in Figure 10. 